\title{
MicroRNAs 21 and 199a-3p Regulate Axon Growth Potential through Modulation of Pten and $m$ Tor mRNAs
}

\author{
Amar N. Kar, ${ }^{1}$ Seung-Joon Lee, ${ }^{1}$ DPabitra K. Sahoo, ${ }^{1}$ Elizabeth Thames, ${ }^{1}$ Soonmoon Yoo, ${ }^{2}$ \\ John D. Houle, ${ }^{3}$ and 1 Jeffery L. Twiss ${ }^{1}$
}

https://doi.org/10.1523/ENEURO.0155-21.2021

${ }^{1}$ Department Biological Sciences, University of South Carolina, Columbia, SC 29208, ${ }^{2}$ Nemours Biomedical Research, Alfred I. duPont Hospital for Children, Wilmington, DE 19803, and ${ }^{3}$ Department of Neurobiology and Anatomy, Drexel University College of Medicine, Philadelphia, PA 19129

\section{Visual Abstract}
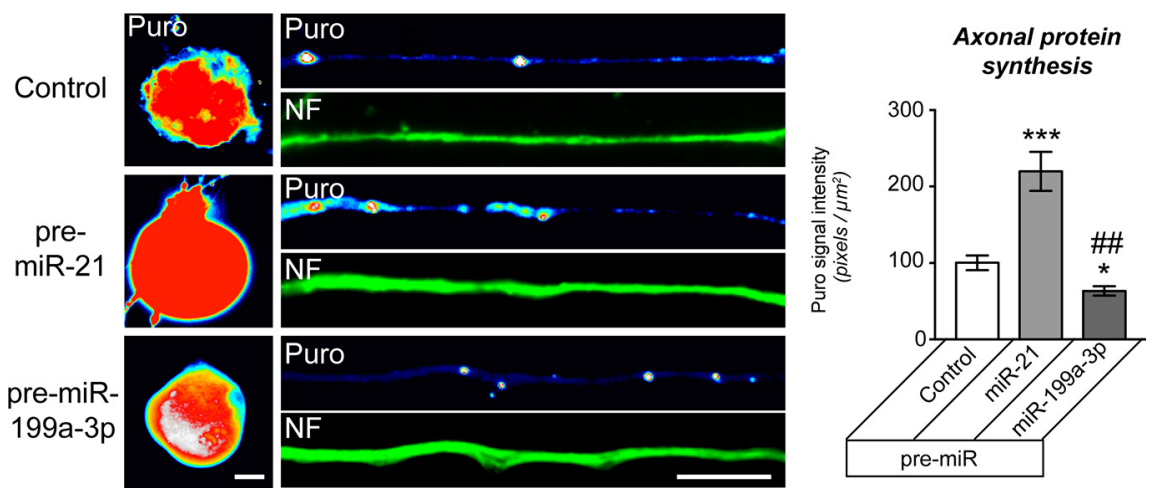

Increased mTOR activity has been shown to enhance regeneration of injured axons by increasing neuronal protein synthesis, while PTEN signaling can block mTOR activity to attenuate protein synthesis. MicroRNAs (miRs) have been implicated in regulation of PTEN and mTOR expression, and previous work in spinal cord showed an increase in miR-199a-3p after spinal cord injury (SCl) and increase in miR-21 in SCl animals that had undergone exercise. Pten mRNA is a target for miR-21 and miR-199a-3p is predicted to target mTor mRNA. Here, we show that miR-21 and miR-199a-3p are expressed in adult dorsal root ganglion (DRG) neurons, and we used culture preparations to test functions of the rat miRs in adult DRG and embryonic cortical neurons. miR-21 increases and miR-199a-3p decreases in DRG neurons after in vivo axotomy. In both the adult DRG and embryonic cortical neurons, miR-21 promotes and miR-199a-3p attenuates neurite growth. miR-21 directly bound to Pten mRNA and miR-21 overexpression decreased Pten mRNA levels. Conversely, miR-199a-3p directly bound to mTor mRNA and miR-199a-3p overexpression decreased mTor mRNA levels. Overexpressing miR-21 increased both overall and intra-axonal protein synthesis in cultured DRGs, while miR-

\section{Significance Statement}

Posttranscriptional regulation of neuronal gene expression has been shown to contribute to axon regeneration after nervous system injury. We show that the PTEN/mTOR pathway is regulated by microRNAs (miRs) after neuronal injury and this modifies the capacity for translation of regeneration-associated genes in primary neurons. 
199a-3p overexpression decreased this protein synthesis. The axon growth phenotypes seen with miR-21 and miR-199a-3p overexpression were reversed by co-transfecting PTEN and mTOR cDNA expression constructs with the predicted $3^{\prime}$ untranslated region (UTR) miR target sequences deleted. Taken together, these studies indicate that injury-induced alterations in miR-21 and miR-199a-3p expression can alter axon growth capacity by changing overall and intra-axonal protein synthesis through regulation of the PTEN/mTOR pathway.

Key words: axonal mRNA; axonal translation; microRNA; PTEN; regeneration; translation

\section{Introduction}

Posttranscriptional regulation of gene expression has proven to be a critical mechanism driving neuronal development, function, and injury responses (Popovitchenko and Rasin, 2017; Bae and Miura, 2020; Dalla Costa et al., 2021). These regulatory mechanisms include processing RNA transcription products in the nucleus, stabilizing or destabilizing mRNAs in the cytoplasm, subcellular localization of mRNAs, and translational regulation. Non-coding microRNAs (miRs) contribute to both stability and translational regulation of mRNAs by binding to specific seed sequences in $3^{\prime}$ untranslated regions (UTRs) of cellular mRNAs. Previous work showed that miR-199a-3p, which was predicted to target mTOR mRNA, is upregulated in spinal cord after a complete transection spinal cord injury (SCI) at thoracic level 10 (T10; Liu et al., 2012). Exercise after $\mathrm{SCl}$ has been shown to alter gene expression leading to increased spinal cord plasticity and recovery of spinal reflexes (Mendell et al., 2001; Ying et al., 2005; Côté et al., 2011), and passive hindlimb exercise after $\mathrm{SCl}$ was shown to attenuate the SCl-induced increase in miR-199a-3p with a concurrent upregulation of miR-21 (Liu et al., 2012). miR-21 has been shown to target Pten mRNA (Xu et al., 2019; Lopez-Leal et al., 2020) and miR199a-3p is predicted to target $m$ Tor mRNA (Liu et al., 2012). PTEN has been implicated in blocking axon regrowth in both the central and peripheral nervous systems (Park et al., 2008; Abe et al., 2010; Christie et al., 2010; Liu et al., 2010). Among the downstream effects of PTEN signaling is attenuation of mTOR activity to decrease mRNA translation (Switon et al., 2017). Thus, it is intriguing to speculate that the changes in miR-199a-3p and

Received April 10, 2021; accepted July 15, 2021; First published July 29, 2021 The authors declare no competing financial interests.

Author contributions: A.N.K., S.Y., and J.L.T. designed research; A.N.K., S.-J.L., P.K.S., E.T., and S.Y. performed research; J.D.H. contributed unpublished reagents/analytic tools; S.-J.L. and P.K.S. analyzed data; A.N.K., J.D.H., and J.L.T. wrote the paper.

This work was supported by National Institutes of Health Grants P01NS055976 (to J.D.H. and J.L.T.), R21-NS099959 (to S.Y.), and R01-NS117821 (to J.L.T.); the South Carolina Spinal Cord Injury Research Fund Grant 2019PD-02 (to P.K.S.); and the Dr. Miriam and Sheldon G. Adelson Medical Research Foundation (J.L.T.).

Acknowledgements: We thank Nitzan Samra for guidance and critical reagents for the mTor $3^{\prime}$ UTR reporter. J.L.T. is the incumbent SmartState Chair in Childhood Neurotherapeutics at the University of South Carolina.

Correspondence should be addressed to Jeffery L. Twiss at twiss@mailbox. sc.edu.

https://doi.org/10.1523/ENEURO.0155-21.2021

Copyright @ 2021 Kar et al.

This is an open-access article distributed under the terms of the Creative Commons Attribution 4.0 International license, which permits unrestricted use, distribution and reproduction in any medium provided that the original work is properly attributed.
miR-21 reported after $\mathrm{SCl}$ and $\mathrm{SCl}$ plus passive hindlimb exercise (Liu et al., 2012) might alter the growth potential of injured neurons. Here, we have asked whether miR-21 and miR-199a-3p are expressed in adult neurons and the mechanisms underlying their effects in neurons.

Adult dorsal root ganglion (DRG) neurons extend axonal projections in culture in the absence of exogenous growth factors and this growth can be increased by a preconditioning injury (Smith and Skene, 1997) and by voluntary exercise (Molteni et al., 2004). These preconditioned neurons rapidly extend axons through translational regulation of mRNAs rather than new gene transcription during the culture period (Twiss et al., 2000). Sciatic nerve injury also preconditions DRG neurons for regeneration after subsequent SCl (Neumann and Woolf, 1999). Here, we confirm that miR-21 promotes axon growth by depletion of Pten mRNA and protein resulting in increased neuronal protein synthesis, and we find that miR-199a-3p attenuates axon growth by depletion of $m$ Tor mRNA and protein resulting in decreased neuronal protein synthesis. Furthermore, miR-199a-3p localizes to axons where it prevents axonal $m$ Tor mRNA translation to decrease axon growth and this is overcome by inhibition of miR-199a-3p. Importantly, we provide evidence that overexpression of miR-21 or inhibition of miR-199a-3p allows both DRG and embryonic cortical neurons to extend axons on the non-permissive substrate chondroitin sulfate proteoglycan (CSPG), indicating that these miRs directly impact the neuron's ability to extend axons over a previously inhospitable terrain.

\section{Materials and Methods}

\section{Animal care and use}

All vertebrate animal experiments were performed under protocols approved by the Institutional Animal Care and Use Committee. Male Sprague Dawley rats (175-250 g) were used for DRG culture and sciatic nerve injury experiments. Isofluorane was used for anesthesia during the peripheral nerve injury procedure. For peripheral nerve injury, anesthetized rats were subjected to a sciatic nerve crush at mid-thigh as described previously (Twiss et al., 2000). Embryonic day (E)18 male and female rat pups were used for cortical neuron cultures. Animals were euthanized using $\mathrm{CO}_{2}$ asphyxiation per approved methods.

\section{Primary neuron culture}

For primary DRG cultures, L4-5 DRG were harvested in Hibernate-A medium (BrainBits) and then dissociated with collagenase (Life Technologies). After centrifugation and washing in DMEM/F12 (Life Technologies), cells were resuspended in DMEM/F12, $1 \times \mathrm{N} 1$ supplement (Sigma), $10 \%$ fetal bovine serum (HyClone), and $10 \mu \mathrm{m}$ cytosine 
arabinoside (Sigma). Dissociated DRGs were plated immediately on poly-L-lysine (Sigma) and laminin (Millipore) coated coverslips or transfected and then plated on coated coverslips for staining and imaging or polyethylene-tetrathalate (PET) membrane (1- $\mu \mathrm{m}$ pores; Corning) inserts for isolation of axons. For CSPG experiments, adult DRGs were cultured on glass bottom dishes (Greiner) that were coated with $10 \mu \mathrm{g} / \mathrm{ml}$ Aggrecan (R\&D Systems) after the laminin/poly-D-lysine coating above.

For cortical neuron cultures, E18 cortices were dissected in Hibernate E (BrainBits) and dissociated using the Neural Tissue Dissociation kit (Miltenyi Biotec). Dissected cortices were incubated in a prewarmed enzyme mix at $37^{\circ} \mathrm{C}$ for $15 \mathrm{~min}$; tissues were then triturated and applied to a $40 \mu \mathrm{m}$ cell strainer. After washing and centrifugation, neurons were seeded onto poly-D-lysine (Sigma) coated coverslips. For the CSPG experiments, cells were seeded on glass bottom dishes (Greiner) coated with $10 \mu \mathrm{g} / \mathrm{ml}$ aggrecan in addition to the poly-Dlysine. NbActive-1 medium (BrainBits) supplemented with $100 \mathrm{U} / \mathrm{ml}$ of penicillin-streptomycin (Life Technologies), 2 mm L-glutamine (Life Technologies), and $1 \times$ N21 supplement (R\&D Systems) was used as culture medium.

For isolation of axons from cell bodies, the lower membrane surface for DRGs cultures in membrane inserts was carefully scraped as previously described (Zheng et al., 2001). The upper membrane surface was used as a "cell body" compartment. These isolates were processed for RNA preparation as outlined below.

For plasmid transfections, dissociated ganglia were pelleted by centrifugation at $100 \times g$ for $5 \mathrm{~min}$ and resuspended in "nucleofector solution" (Rat Neuron Nucleofector kit; Lonza); 2-5 $\mu \mathrm{g}$ plasmid was electroporated using an AMAXA Nucleofector apparatus (program SCN-8; Lonza). For overexpression or inhibition of miRs, $50 \mathrm{~nm}$ of precursormiRNA (pre-miR) or inhibitor anti-miR were transfected using DharmaFECT 3 reagent (Dharmacon). Scrambled precursor and anti-miR oligomers were used as negative controls. RT droplet digital PCR (ddPCR) and immunoblotting were used to test levels of the endogenous miRNA targets (see below).

\section{DNA constructs}

Pre-miRNA, biotinylated miRs, inhibitor anti-miR, and control RNA oligomers were purchased from Exiqon. The myc-tagged mTOR (wild-type) and kinase dead mTOR mutant (mTOR-Kdm) expression constructs were purchased from Addgene (plasmids \#1861 and \#8482, respectively). All fluorescent reporter constructs for analyses of RNA translation were based on mCherry plasmid with myristoylation element ( $\mathrm{mCh}^{\text {MYR}}$; Vuppalanchi et al., 2012). Reporter constructs containing $5^{\prime}$ and $3^{\prime}$ UTRs of rat Nrn1, Gap43,

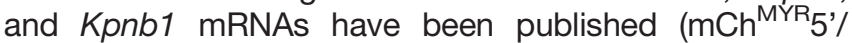
3'nrn1 or $\mathrm{mCh}^{\mathrm{MYR}} 5^{\prime} / 3^{\prime} \mathrm{kpnb} 1$, or $\mathrm{mCh}^{\mathrm{MYR}} 5^{\prime} / 3^{\prime}$ gap43; Sahoo et al., 2018). For mTOR, the rat $3^{\prime}$ UTR was subcloned by PCR from previously published UTR constructs (Terenzio et al., 2018), and inserted directly downstream of the stop codon of $\mathrm{mCh}^{\mathrm{MYR}}$ sequence in a vector that included 5' UTR of rat Camkll mRNA (mCh ${ }^{\mathrm{MYR}_{5}}$ 'camKII/ 3'mtor; Vuppalanchi et al., 2012). Rat PTEN protein coding sequence was generated from a rat DRG cDNA library. Using PCR primers with restriction enzyme sites (EcoRI and Sall), PTEN coding sequence was amplified with high fidelity polymerase (Primer Star HS, Takara) and inserted into pFLAG-CMV2 (Sigma) using EcoRI/Sall sites. All UTR reporter and cDNA expression constructs were confirmed by sequencing.

\section{RNA isolation and PCR analyses}

RNA was isolated from immunoprecipitates and cultures using the RNeasy Micro isolation kit (QIAGEN). Fluorimetry with Ribogreen (Invitrogen) was used to determine RNA quantities. For analyses of total RNA levels and inputs for miR-mRNA co-precipitation analyses, RNA yields were normalized across samples before reverse transcription using Sensifast (Bioline). For quantitating mRNAs co-precipitating with biotinylated miRs (see below), equal proportions of each precipitation were used for reverse transcription with Sensifast. RT products were processed for ddPCR using Evagreen (Bio-Rad) with transcript specific primers (Integrated DNA Tech; sequences available on request). ddPCR reactions were read on a QX200 droplet reader (Bio-Rad).

For miR quantification, tissues and cultures were lysed and total RNA was isolated using the miRVana miRNA Isolation kit (QIAGEN) according to manufacturer's recommendations. Ribogreen assay was performed to determine the RNA quantity in each sample. Equal amounts of total RNA were reverse transcribed using the miRCURY LNA miRNA RT kit (QIAGEN) according to manufacturer's protocol. After RT, the miRNAs were detected using miRspecific primers (Exiqon) by ddPCR with Evagreen detection reagent (Bio-Rad) and QX200 droplet reader.

\section{miR-mRNA coprecipitation}

Biotinylated miR mimics (100 nM) were transfected into DRG neurons as above; 16-24 $\mathrm{h}$ after transfection, cultures were lysed in $20 \mathrm{~mm}$ Tris- $\mathrm{HCl}(\mathrm{pH} 7.5), 10 \mathrm{~mm} \mathrm{KCl,} 5$ $\mathrm{mm} \mathrm{MgCl}$, and $0.3 \% \mathrm{NP}-40$ supplemented with protease and RNase inhibitors. Lysates were cleared by centrifugation for $10 \mathrm{~min}$ at $4^{\circ} \mathrm{C}, 18,000 \times \mathrm{g}$. Supernatants were collected, and equal volume of the supernatant were incubated with preequilibrated Streptavidin M280 Magnetic Beads (Life Technologies) overnight an end-over-end rotator at $4^{\circ}$ C; $10 \%$ of the lysate was processed for RNA purification using the miRVana miRNA isolation kit, as described earlier for input. After incubation, the beads were washed in lysis buffer and then processed for RNA isolation as outlined above.

\section{Immunoblotting}

Adult rat DRG cultures (3 d in vitro, $\sim 60,000$ neurons/ well) were lysed in $50 \mathrm{~mm}$ Tris- $\mathrm{HCl}, 10 \mathrm{~mm}$ EDTA, $0.1 \mathrm{~mm}$ DTT, $1 \%$ SDS, and $1 \times$ Mini EDTA-free Proteinase Inhibitor (Roche) $36 \mathrm{~h}$ after transfections. Samples were denatured by boiling at $95^{\circ} \mathrm{C}$ for $5 \mathrm{~min}$. Lysates were cleared of debris by centrifugation at $15,000 \times g$ for $15 \mathrm{~min}$ at $4^{\circ} \mathrm{C}$ and then normalized for protein content using BCA assay (Bio-Rad). Normalized protein lysates were fractionated on $8 \%$ SDS/ 
PAGE gels and transferred to PVDF membranes (GE Healthcare). After blocking in 5\% non-fat dried milk (Bio-Rad) diluted in Tris-buffered saline with 1\% Tween 20 (TBST), membranes were probed overnight at $4^{\circ} \mathrm{C}$ with rabbit anti-mTOR (1:1000; Abcam), rabbit antiPTEN (1:1000, Abcam), or rabbit anti-GAPDH (1:2000; Cell Signaling Technology) antibodies diluted in blocking buffer. Blots were washed in TBST and then incubated with horseradish peroxidase (HRP)-conjugated anti-rabbit IgG (1:2000; Cell Signaling Technology) diluted in blocking buffer for $1 \mathrm{~h}$ at room temperature. Blots were washed in TBST and signals detected with ECL Prime (GE Healthcare).

\section{Puromycinylation assay}

To visualize newly synthesized proteins in cultured DRG neurons, we used the Click-iT Plus OPP Protein Synthesis Assay kit per manufacturer's instructions (Invitrogen). Briefly, 3 d DRG cultures were incubated with $20 \mu \mathrm{M}$ O-propargyl-puromycin (OPP) for $30 \mathrm{~min}$ at $37^{\circ} \mathrm{C}$. OPP-labeled proteins were detected by crosslinking with Alexa Fluor 594 picolyl azide molecule. Coverslips were then mounted with Prolong Gold Antifade (Invitrogen) and imaged with Leica DMI6000 epifluorescent microscope as above. ImageJ (National Institutes of Health) was used to quantify the incorporated puromycinylation signals in distal axons and cell bodies.

\section{Immunofluorescence}

All steps occurred at room temperature unless specified otherwise. DRG and cortical neuron cultures were rinsed in PBS and then fixed in 4\% paraformaldehyde in PBS for 20 min. Coverslips were then rinsed in PBS and then permeabilized in PBS plus $0.5 \%$ Tween 20. After rinsing in PBS again, coverslips were blocked in 5\% donkey serum in PBS plus $0.1 \%$ Tween 20 (PBST) for $1 \mathrm{~h}$ followed by overnight incubation at $4^{\circ} \mathrm{C}$ in primary antibodies diluted in blocking buffer. Mouse RT-97 anti-neurofilament (NF; 1:2000; Development Hybridoma Studies Bank) and mouse anti-FLAG (1:2000; Sigma) were used for primary antibodies. Coverslips were rinsed in PBS and then incubated with secondary antibodies diluted in blocking buffer for $1 \mathrm{~h}$. Secondary antibodies consisted of FITC-conjugated goat-anti-mouse IgG antibody and CY5 conjugated anti-rabbit (both at 1:400; Jackson ImmunoResearch). After rinsing in PBS followed by distilled $\mathrm{H}_{2} \mathrm{O}$, samples were mounted with Prolong Gold antifade.

\section{Axon growth analyses}

For neurite and axon outgrowth, images from 48- to 60h DRG or cortical neuron cultures were acquired using the ImageXpress Micro High-Content Imaging System (Molecular devices). These images were analyzed for neurite outgrowth using WIS-Neuromath (Rishal et al., 2013). Neurites were visualized using NF immunofluorescence. Neurite lengths were measured for at least 75 neurons per treatment group per culture preparation.

\section{Fluorescence recovery after photobleaching (FRAP)}

FRAP was used to evaluate axonal protein synthesis using diffusion-limited mCherry ${ }^{\mathrm{MYR}}$ reporters as described with minor modifications (Yudin et al., 2008). DRG neurons were co-transfected with $\mathrm{mCh}^{\mathrm{MYR}}{ }_{5}$ 'camKII/ 3'mtor, mCh ${ }^{\mathrm{MYR}} 5$ '/3'nrn1, mCh ${ }^{\mathrm{MYR}} 5$ '/3'kpnb1, or mCh ${ }^{\mathrm{MYR}} 5$ '/ 3'gap43 plus pre-miR-199a-3p or control RNA. Cells were maintained at $37^{\circ} \mathrm{C}, 5 \% \mathrm{CO}_{2}$ during imaging sequences. $514 \mathrm{~nm}$ laser line on Leica SP8X confocal microscope was used to bleach $\mathrm{mCh}^{\mathrm{MYR}}$ signals (Argon laser at $70 \%$ power, pulsed every $0.82 \mathrm{~s}$ for 80 frames). Pinhole was set to 3 airy units to ensure full thickness bleaching and acquisition $(63 \times)$ 1.4 NA oil immersion objective). Before photobleaching, neurons were imaged every $60 \mathrm{~s}$ for $2 \mathrm{~min}$ to acquire baseline fluorescence for the region of interest (ROI). The same excitation and emission parameters were used to assess recovery over $15 \mathrm{~min}$ postbleach with images acquired at $30 \mathrm{~s}$ intervals. To determine whether fluorescence recovery in axons because of translation, DRG cultures were treated with 100$\mu \mathrm{m}$ anisomycin (Sigma) for $30 \mathrm{~min}$ before photobleaching. To evaluate whether fluorescence recovery was mTOR-dependent, DRG cultures were treated with 10-nm rapamycin (Sigma) for $30 \mathrm{~min}$ before photobleaching.

Fluorescent intensities in the ROls were calculated using Leica LASX software. For normalizing across experiments, fluorescence intensity value at $t=0 \mathrm{~min}$ postbleach from each image sequence was set as $0 \%$ and prebleach values at $100 \%$. The percentage of fluorescence recovery at each time point after photobleaching was then calculated by normalizing relative to the prebleach fluorescence (Vuppalanchi et al., 2010).

\section{Statistical analyses}

Prism (GraphPad) was used for all statistical analyses. One-way ANOVA with Tukey's post hoc correction was used in all experiments unless specified. For the statistical analyses of FRAP studies two-way ANOVA with Tukey's post hoc test was used; $p<0.05$ was considered significant. Numbers of experimental replicates are indicated in figure legends.

\section{Results}

\section{DRG levels of miR-21 and miR-199a-3p change on axotomy}

Although spinal cord levels of miR-199a-3p were shown to be increased after SCl and those of miR-21 increased when $\mathrm{SCl}$ animals are subjected to exercise (Liu et al., 2012; Li et al., 2020), the cellular source of these miRs was not clear from these previous publications. Moreover, the predictions that miR-21 would target neuronal Pten mRNA and miR-199a-3p would target neuronal $m$ Tor mRNA were not specifically tested. We addressed these knowledge gaps using rat DRG neurons that can be cultured from adult animals and show increased regeneration in the injured spinal cord after $\mathrm{SCl}$ when they are preconditioned by a peripheral nerve crush injury (Neumann and Woolf, 1999; Neumann et al., 2002). We initially used fluorescence in situ hybridization (FISH) to test for expression of these miRs in the DRGs in vivo. With locked nucleic 

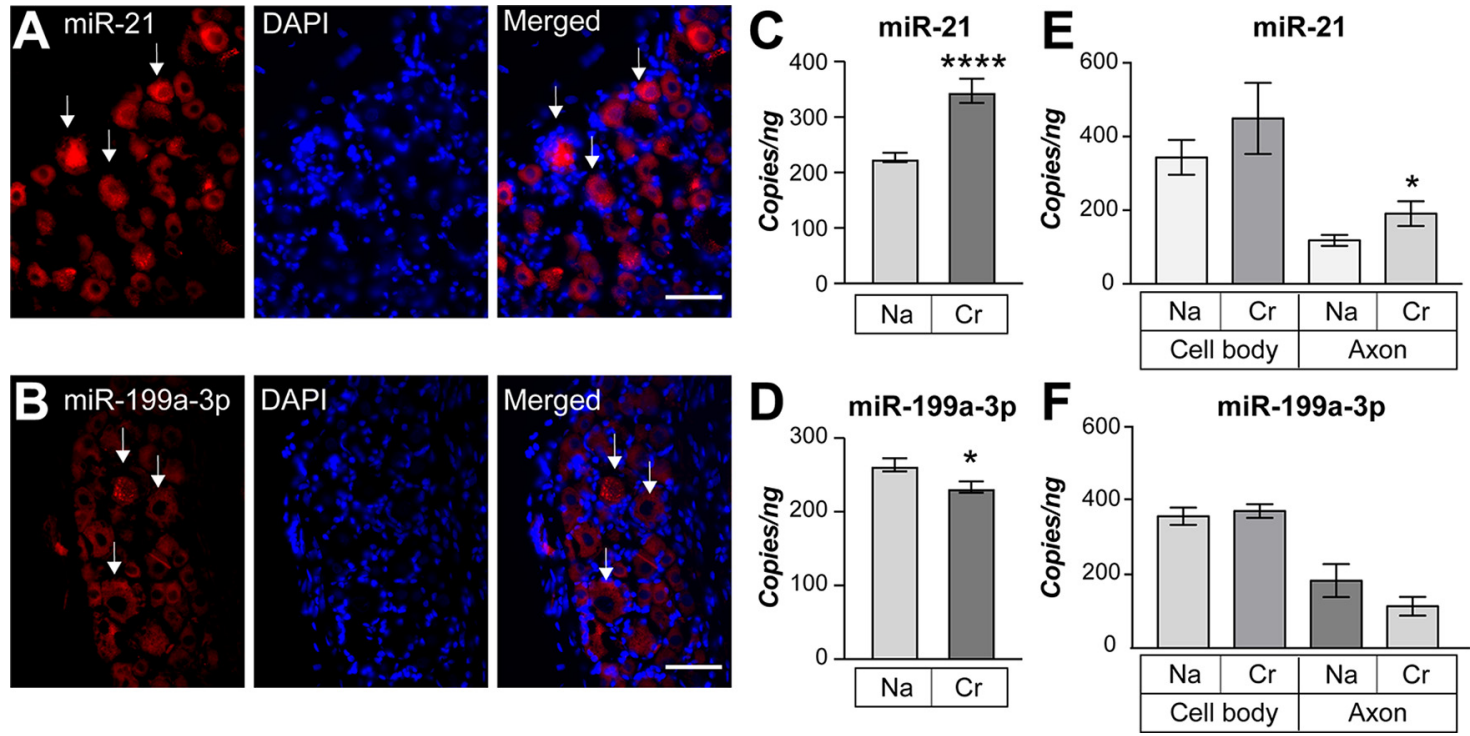

Figure 1. miR-21 and miR-199a-3p are expressed in adult DRG neurons. $\boldsymbol{A}, \boldsymbol{B}$, Exposure matched images for endogenous miR-21 $(\boldsymbol{A})$ or miR-199a-3p (B) plus DAPI in DRG sections derived from adult naive rats. Arrows indicate miR-expressing DRG neurons. Scale bar: $50 \mu \mathrm{m}$. $\boldsymbol{C}, \boldsymbol{D}$, Quantification of mature miR-21 $(\boldsymbol{C})$ and miR-199a-3p (D) levels in dissociated DRG neurons derived from naive $(\mathrm{Na})$ and 7 -d injury-conditioned $(\mathrm{Cr})$ animals by RT-ddPCR analyses shown as average copies \pm SEM per ng of RNA input. $\boldsymbol{E}$, $\boldsymbol{F}$, Quantification of miR-21 $(\boldsymbol{E})$ and miR-199a-3p $(\boldsymbol{F})$ levels in cell bodies and axons isolated from naive and injury conditioned DRG neurons is shown as average copies \pm SEM per ng of RNA input $\left(N \geq 4\right.$ for $\boldsymbol{C}-\boldsymbol{F} ;{ }^{*} p \leq 0.05,{ }^{* * * *} p \leq 0.001$ vs naive axon by Student's $t$ test vs naive axon by Student's $t$ test).

acid (LNA) probes specific for mature miRs, we see clear cytoplasmic signals for both miR-21 and miR-199a-3p in the DRG neurons (Fig. $1 A, B$ ). Unfortunately, the LNA FISH signals were not amenable to quantification, so we used RNA extracted from DRG culture preparations to gain a better understanding of the levels of these miRs in the DRG neurons. DRG neurons that have been preconditioned by an in vivo axotomy show rapid axonal outgrowth in culture that is translationally regulated (Smith and Skene, 1997; Twiss et al., 2000). By reverse transcriptase coupled ddPCR (RTddPCR) there was a significant increase in miR-21 and decrease in miR-199a-3p levels in the L4-6 DRGs harvested ipsilateral to a sciatic nerve crush lesion performed $7 \mathrm{~d}$ before culture (Fig. 1C,D).miRs have been shown to localize into sensory axons and their levels can dynamically change during regeneration (Kim et al., 2015; Phay et al., 2015). Since translation of mRNAs in axons has been shown to facilitate regeneration in the PNS (Smith et al., 2020), we asked whether these miRs localize into axons and whether their axonal levels change with the injury conditioning. For this, naive and 7-d injury-conditioned L4-6 dissociated DRGs were cultured on a porous membrane for isolation of axons (Willis and Twiss, 2010). Interestingly, the axon preparations from injury-conditioned DRGs showed significantly increased miR-21 levels (Fig. 1E). The axonal levels of miR199a-3p were decreased, but this did not reach statistical significance (Fig. 1F). Neither miR-199a-3p nor miR-21 levels significantly changed in cell body preparations with injury conditioning (Fig. 1E,F). Thus, consistent with previous observations in mouse DRGs (Cheng et al., 2015), we find that rat DRG neurons express both miR-21 and miR-199a-3p but also that these expression of these miRs changes with injury conditioning and both miRs localize to axons.

\section{miR-21 and miR-199a-3p target neuronal Pten and $m$ Tor mRNAs}

We next asked whether miR-21 and miR-199a-3p target the predicted Pten and $m$ Tor mRNAs in the adult DRG cultures. For this, we transfected biotinylated miR-21 and miR-199a-3p mimics into naive adult DRG cultures and used RT-ddPCR to determine whether the mRNAs were bound in streptavidin (SA) precipitations. Pten mRNA was highly enriched in the miR-21 precipitates as compared with the scrambled control RNA SA precipitations (Fig. $2 A)$. In contrast, mTor mRNA was highly enriched in the miR-199a-3p compared with scrambled control RNA SA precipitations (Fig. 2B). Importantly, mTor mRNA was not significantly different from control RNA in the miR-21 precipitates and Pten mRNA was not significantly different from control RNA in the miR-199a-3p precipitates (Fig. $2 A, B)$.

We next transfected DRG cultures with miR precursor RNAs (pre-miRs) to exogenously elevate miR-21 and miR-199a-3p levels versus the corresponding anti-miRs to inhibit functions of endogenous miR-21 and miR-199a$3 p$ in DRG cultures. As expected, transfections with pre-miR-21 and pre-miR-199a-3p significantly increased levels of the mature miR-21 and miR-199a-3p, respectively (Fig. $2 C, D$ ). Pre-miR-21 transfection significantly decreased endogenous Pten mRNA and protein levels (Fig. $2 E-G)$. Pre-miR-199a-3p transfection significantly decreased endogenous $m$ Tor mRNA and protein levels (Fig. $2 /-K)$. Conversely blocking the functions of endogenous miR-21 using a specific anti-miR increased endogenous Pten mRNA levels (Fig. 2H), while anti-miR-199a-3p increased endogenous $m$ Tor mRNA levels (Fig. 2L). Taken together, these data point to Pten and mTor mRNAs as 
A Pten mRNA

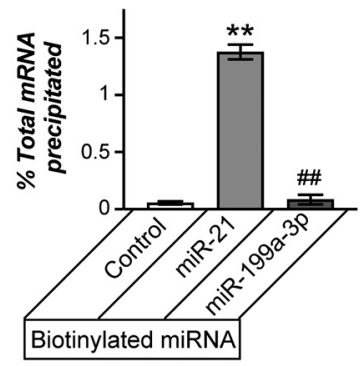

E

E Pten mRNA
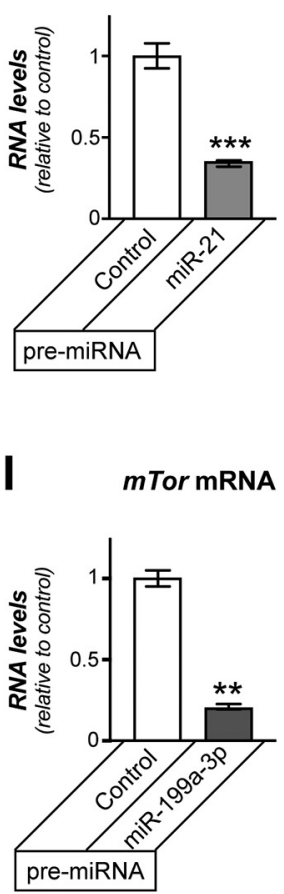

B
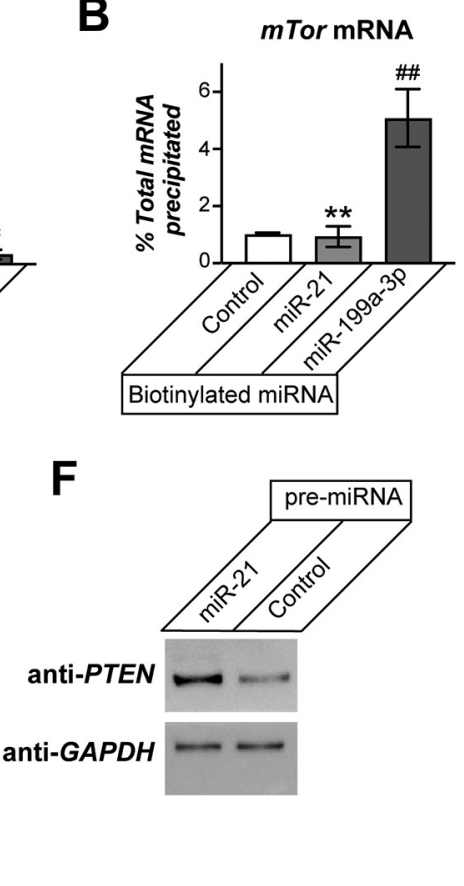

J

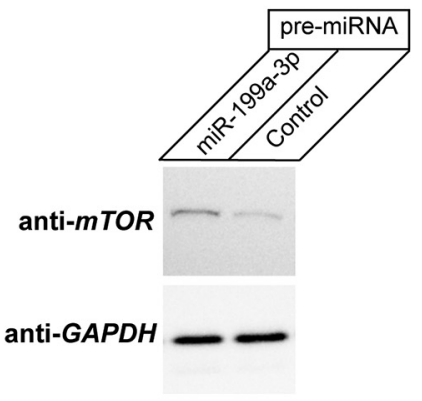

C
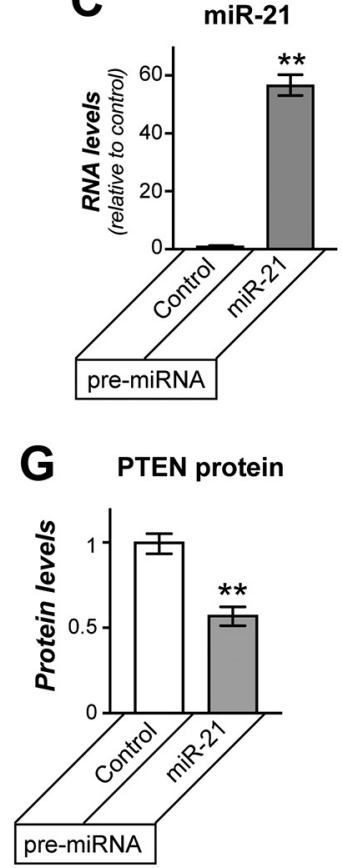

K mTOR protein

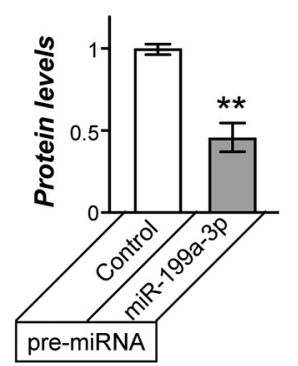

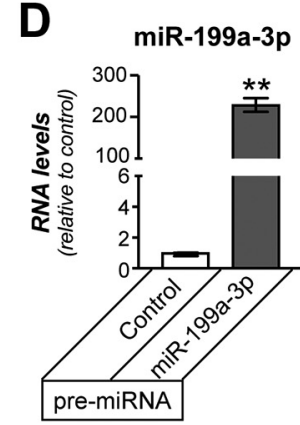

H

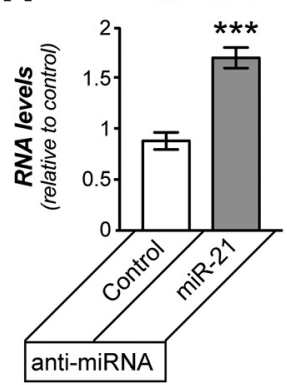

Figure 2. miR-21 and miR-199a-3p target $m$ Tor and Pten mRNAs. $\boldsymbol{A}, \boldsymbol{B}, \mathrm{RTddPCR}$ for Pten $(\boldsymbol{A})$ and $m$ Tor $(\boldsymbol{B}) \mathrm{mRNAs}$ from precipitations of 3'biotinylated miR-21, miR-199a-3p or scrambled RNA (control) transfected DRG neurons is shown as average relative to total input mRNA \pm SEM $\left(N \geq 4 ;{ }^{* *} p \leq 0.01\right.$ vs control and $\# \# p \leq 0.01$ vs miR-21 by one-way ANOVA with pair-wise comparison and Tukey's post hoc tests). C, $\boldsymbol{D}$, Transfection of precursor miR-21 and miR-199a-3p (pre-miR-21 and pre-miR-199a-3p, respectively) into DRG neurons increased levels of mature miR-21 (C) and miR-199a-3p levels $(\boldsymbol{D})$. $\boldsymbol{E}-\boldsymbol{G}$, DRG cultures transfected with pre-miR21 showed decrease in Pten mRNA by RTddPCR $(\boldsymbol{E})$ and PTEN protein by immunoblotting $(\boldsymbol{F}, \boldsymbol{G})$. $\boldsymbol{F}$, Representative immunoblot for PTEN protein, with GAPDH immunoblot showing relatively equal loading between lanes. $\boldsymbol{E}, \boldsymbol{K}$, Average \pm SEM. $\boldsymbol{H}$, Anti-miR-21 transfection in adult DRG neuron cultures increases levels of Pten mRNA. I-K, DRG cultures transfected with pre-miR-199a-3p showed decrease in mTor mRNA by RTddPCR $(\boldsymbol{I})$ and mTOR protein by immunoblotting $(\boldsymbol{J}, \boldsymbol{K})$. $\boldsymbol{F}$, Representative immunoblot for mTOR protein, with GAPDH immunoblot showing relatively equal loading between lanes. $\boldsymbol{I}, \boldsymbol{K}$, Average values $\pm \mathrm{SEM}$. $\boldsymbol{L}$, Anti-miR199a-3p transfection in adult DRG neuron cultures increases levels of $m$ Tor mRNA ( $N=4$ for $\boldsymbol{E}, \boldsymbol{H}-\boldsymbol{J} ; \boldsymbol{N}=3$ for $\boldsymbol{G}, \boldsymbol{K}$; ${ }^{*} p \leq 0.01$ and ${ }^{* * *} p \leq 0.005$, vs control by Student's $t$ test).

direct targets for miR-21 and miR-199a-3p, respectively, in adult DRG neurons.

\section{Modulation of miR-21 and miR199a-3p levels alters axon outgrowth in DRG neurons}

Considering the miR expression and mRNA target studies above together with the reported roles of PTEN to mTOR pathway in axon growth (Park et al., 2008; Abe et al., 2010; Sun et al., 2011; Duan et al., 2015; Chen et al.,
2016; Terenzio et al., 2018), we asked whether manipulation of miR-21 and miR-199a-3p levels and function might affect axon growth in the DRG neurons. DRG neurons transfected with pre-miR-21 showed significantly increased axon lengths, while those transfected with pre-miR-199a-3p showed significantly decreased axon lengths (Fig. $3 A, C$ ). The pre-miR-21 transfected neurons also showed significantly decreased axon branching (Fig. $3 A, D)$. In contrast, DRGs transfected with anti-miR21 showed reduced axon lengths and those transfected 
A
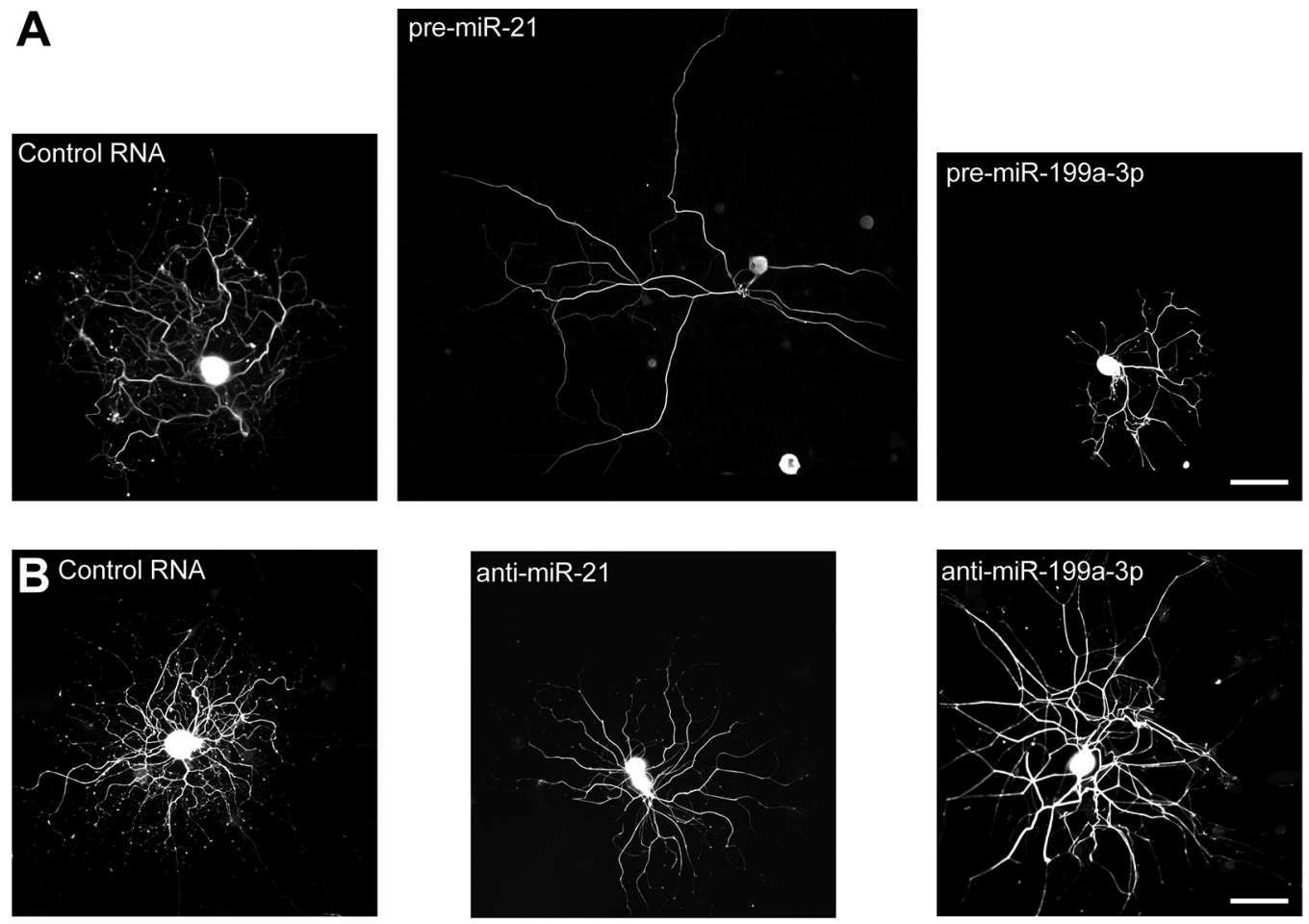

C

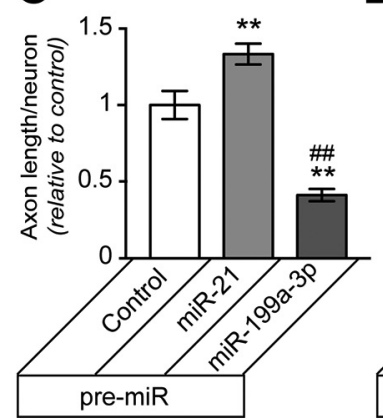

D

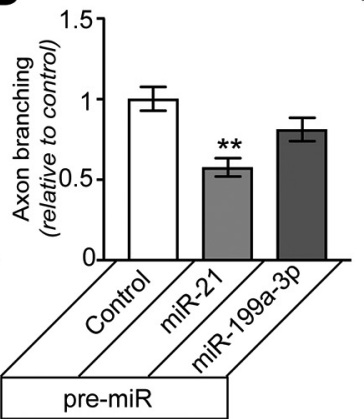

E

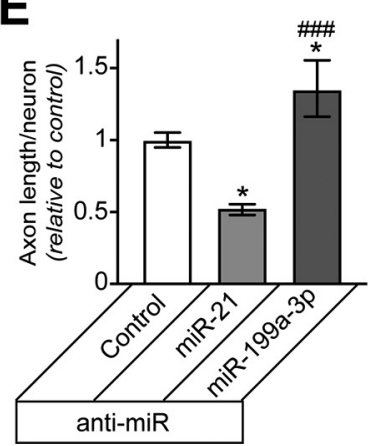

$\mathbf{F}$

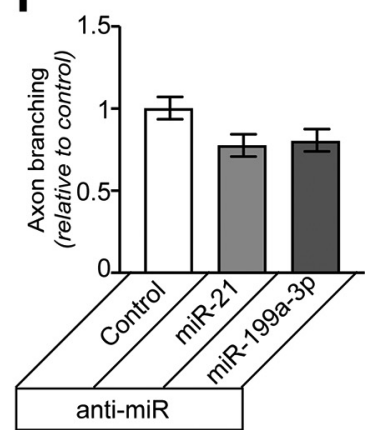

Figure 3. Changing miR-21 and miR-199a-3p levels alters axon growth in DRG neurons. $\boldsymbol{A}$, $\boldsymbol{B}$, Representative immunofluorescent images for NF in rat DRG cultures transfected with indicated pre-miRs versus scrambled RNA (control; $\boldsymbol{A}$ ) or anti-miRs versus scrambled RNA $(\boldsymbol{B})$ are shown. $\boldsymbol{C}, \boldsymbol{D}$, Total axon length per neuron $(\boldsymbol{C})$ and axon branching $(\boldsymbol{D})$ are shown for DRG neurons transfected with pre-miRs as in $\boldsymbol{A}$ as average \pm SEM relative to scrambled RNA transfection. $\boldsymbol{E}, \boldsymbol{F}$, Total axon length per neuron $(\boldsymbol{E})$ and axon branching $(\boldsymbol{F})$ are shown DRG neurons transfected with anti-miRs as in $\boldsymbol{B}$ as average \pm SEM relative to control $(n \geq 100$ neurons each over 3 experimental replicates for $\boldsymbol{C}-\boldsymbol{F} ;{ }^{* *} p \leq 0.01$ vs control and \#\#p $\leq 0.01$, \#\#\#p $\leq 0.005$ comparing between pre-miRs and anti-miRs using one-way ANOVA with pair-wise comparison and Tukey's post hoc tests). Scale bars: $100 \mu \mathrm{m}(\boldsymbol{A}, \boldsymbol{B})$.

with anti-miR-199a-3p showed increased axon lengths (Fig. 3B,E). The anti-miR transfections has no significant effect on axon branching (Fig. 3B,F).

The increased axon length and decreased axon branching seen with miR-21 overexpression is reminiscent of the "elongating" growth morphology that Smith and Skene (1997) described for injury-conditioned DRG neurons (Smith and Skene, 1997). With the elevation of endogenous miR-21 and decrease in endogenous miR-199a-3p in injury-conditioned neurons seen in Figure 1, we asked whether manipulating miR21 and miR-199a-3p levels and function affects axon growth from injury-conditioned DRG neurons. Seven- day injury-conditioned DRG cultures transfected with pre-miR-21 showed no change in axon length compared with control, but the pre-miR-199a-3p transfected neurons showed significantly decreased axon lengths (Fig. 4A,C). In contrast, anti-miR-21 transfection significantly decreased axon length in the injuryconditioned DRG neurons, but anti-miR-199a-3p had no effect (Fig. 4B,D). Neither pre-miRs nor anti-miRs had any significant effect on axon branching (Fig. 4E, $F)$. Taken together with the miR expression data shown for naive versus injury-conditioned neurons shown in Figure $1 A$, these results point to the increase in miR-21 and decrease in miR-199a-3p seen in the injury- 

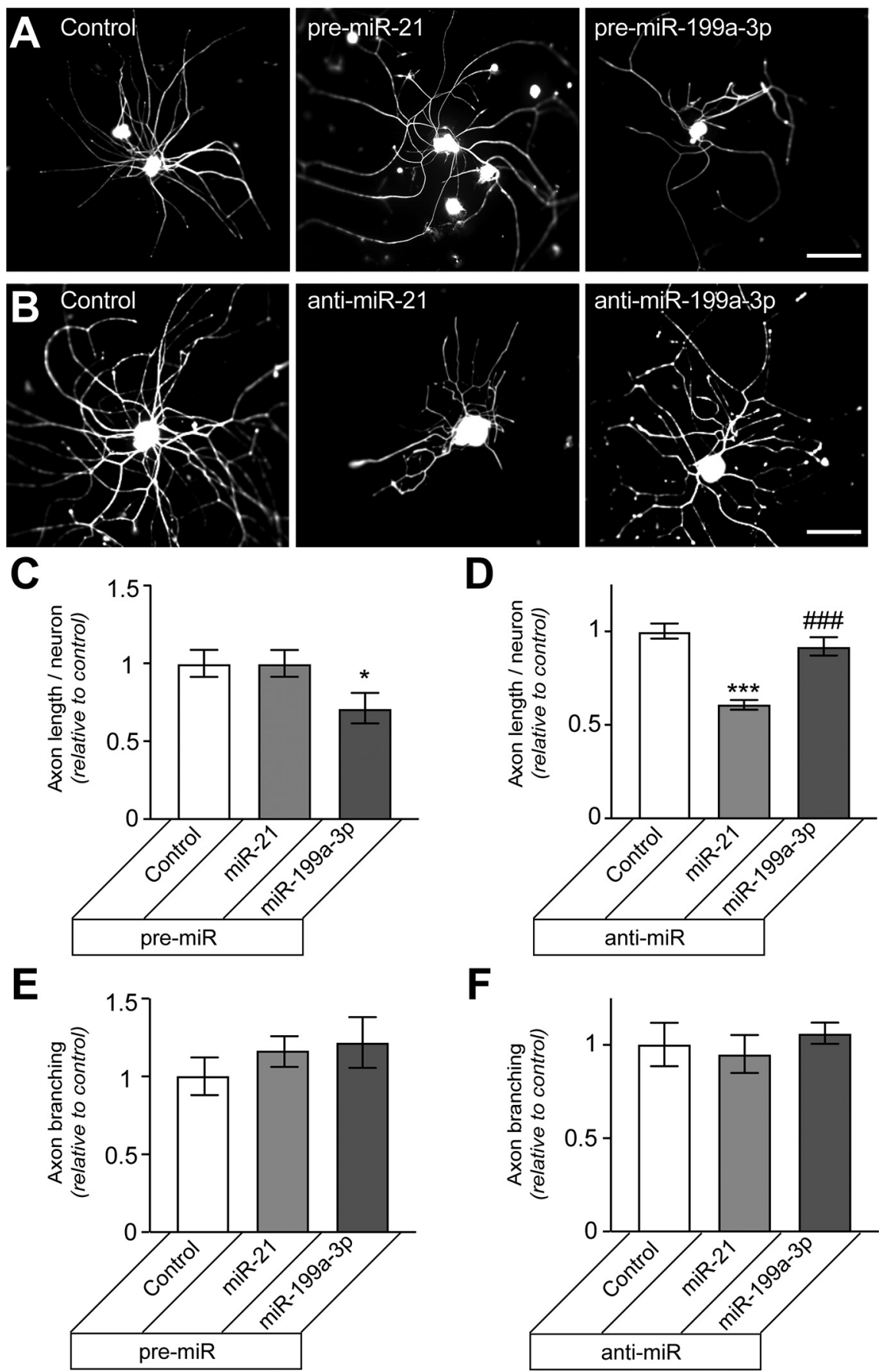

Figure 4. Overexpressing miR-199a-3p or blocking miR-21 decrease axon growth in injury-conditioned DRG neurons. $\boldsymbol{A}$, $\boldsymbol{B}$, Representative immunofluorescent images for NF in rat L4-5 DRG neurons that were injury conditioned by sciatic nerve crush $7 \mathrm{~d}$ before culture are shown. $\boldsymbol{A}$, DRGs transfected with indicated pre-miRs versus scrambled RNA (control). $\boldsymbol{B}$, DRGs transfected with anti-miRs versus scrambled RNA. C, $\boldsymbol{D}$, Total axon length per neuron is shown for the injury conditioned DRG neurons that were transfected with the indicated pre-miRs $(\boldsymbol{C})$ or anti-miRs $(\boldsymbol{D})$ as average \pm SEM relative to control. $\boldsymbol{E}, \boldsymbol{F}$, Axon branching for injury-conditioned neurons transfected with the indicated pre-miRs $(\boldsymbol{E})$ or anti-miRs $(\boldsymbol{F})$ shown as average \pm SEM relative to control $(n \geq 150$ neurons each over 3 experimental replicates for $\boldsymbol{C}-\boldsymbol{F} ;{ }^{*} p \leq 0.05$ and ${ }^{\star \star *} p \leq 0.005$ vs control and \#\#\#p $\leq .005$ comparing between pre-miRs and anti-miRs using one-way ANOVA with pair-wise comparison and Tukey's post hoc tests; no significant differences were for axon branching). Scale bars: $100 \mu \mathrm{m}(\boldsymbol{A}, \boldsymbol{B})$.

conditioned DRG cultures as drivers for the elongating axon growth from these neurons. Considering that axon growth from injury-conditioned neurons requires translation of an mRNA cohort that exists at the time of culture (Twiss et al., 2000), these data not unsurprisingly raise the possibility that $\mathrm{mTOR}$ contributes to the translation of those mRNAs needed for the rapid axon elongation from injury-conditioned neurons. 
A

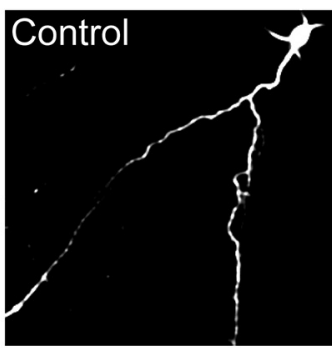

C

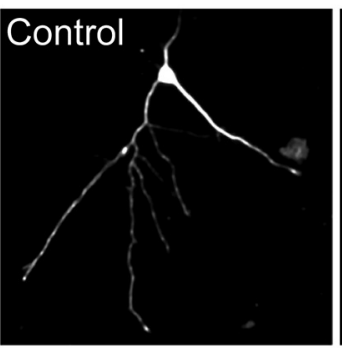

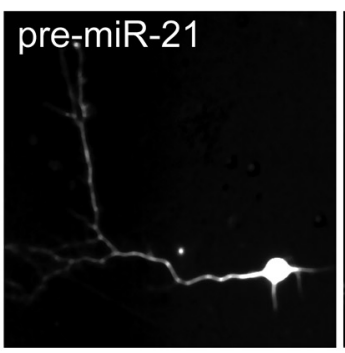

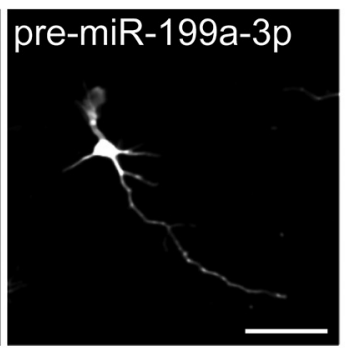

B

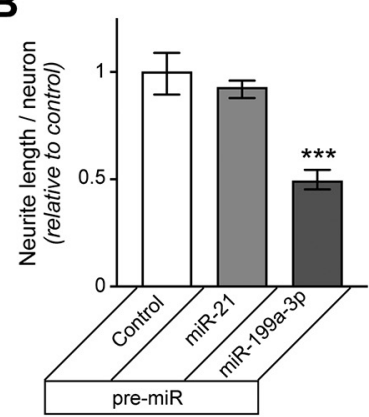

D
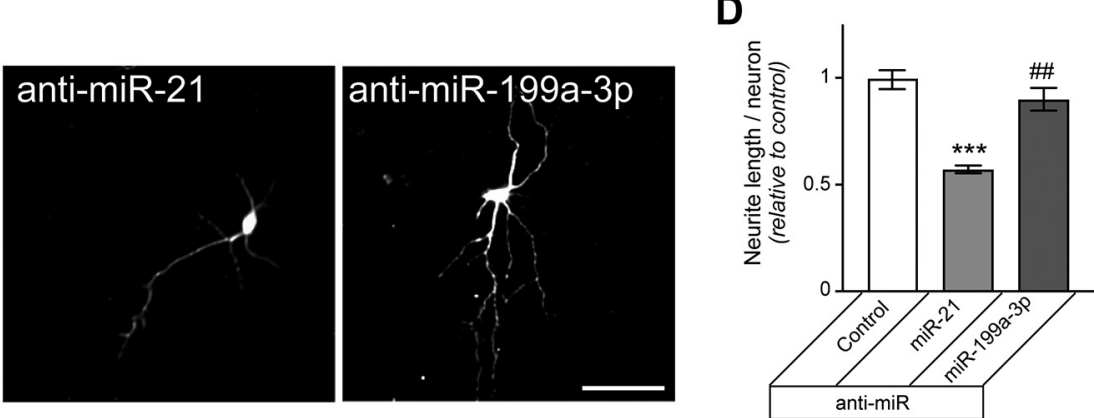

Figure 5. miR-21 and miR-199a-3p levels alter neurite growth in cortical neurons. $\boldsymbol{A}, \boldsymbol{B}$, Representative immunofluorescent images for NF-stained E18 cortical neurons transfected with indicated pre-miRs versus scrambled RNA (control) are shown in $\boldsymbol{A}$. Total neurite length per transfected neuron is shown in $\boldsymbol{B}$ as average \pm SEM relative to control $(n \geq 95$ each over 3 experimental replicates; ${ }^{* \star *} p \leq 0.0001$ vs control and pre-miR-21 by one-way ANOVA with pair-wise comparison and Tukey's post hoc tests). $\boldsymbol{C}$, $\boldsymbol{D}$, Representative NF immunofluorescent images for E18 cortical neurons transfected with indicated anti-miRs versus or scrambled RNA are shown in $\boldsymbol{C}$. Total neurite length per transfected neuron is shown in $\boldsymbol{D}$ as average \pm SEM relative to control $(N \geq 95$ each over 3 experimental replicates for $\boldsymbol{B}, \boldsymbol{D} ;{ }^{* * *} p \leq 0.001$ vs control and $\# \# p \leq 0.005$ vs anti-miR-21 by one-way ANOVA with pair-wise comparison and Tukey's post hoc tests). Scale bars: $50 \mu \mathrm{m}(\boldsymbol{A}, \boldsymbol{B})$.

\section{Modulation of miR-199a-3p and miR-21 levels alters neurite outgrowth from cortical neurons}

Since the PTEN-mTOR pathway has also been shown to affect axon regeneration after optic nerve and corticospinal tract injuries (Park et al., 2008; Liu et al., 2010), we tested whether miR-21 and miR-199a-3p might also affect growth potential of CNS neurons. For this, dissociated E18 cortical neurons were transfected with pre-miRs or anti-miRs at the time of culture and then assessed for neurite growth at $48-60 \mathrm{~h}$ later. As this culture duration precedes complete development of axonal versus dendritic polarity (Craig and Banker, 1994), we assessed overall neurite growth rather than axon growth in these cultures. The cortical neurons transfected with pre-miR-199a-3p showed significantly decreased neurite lengths, but transfection with pre-miR-21 had no effect (Fig. $5 A, B$ ). In contrast, transfection with anti-miR-21 decreased axon growth in the cortical neurons, while transfection with anti-miR199a-3p had no effect (Fig. 5C,D). These results indicate that miR-21 and miR-199a-3p levels influence the intrinsic growth ability of cortical neurons. Moreover, these findings suggest that similar to the injury-conditioned neurons, miR-21 is at saturating levels compared with miR-199a-3p in these embryonic cortical neurons.

Although the Houle lab used female rats for the SCI studies where miR-21 and miR-199a-3p levels were altered (Liu et al., 2012), we used male rats for the DRG cultures shown in Figures 1-4. Thus, we could not exclude the possibility of sexually dimorphic functional responses to miR-199a-3p and miR-21. Since the embryonic cortical neurons used here were from both male and female, we reasoned that dimorphic responses would generate two populations of neurons in the cortical cultures, ones whose neurite lengths were affected by the miR manipulations and a second where lengths were unaffected or affected in the opposite direction. To address this possibility, we evaluated the distribution of neurite lengths in the pre-miR and anti-miR transfected cortical neurons. Uni-modal distribution of neurite lengths were seen in pre-miR-21, pre-miR-199a-3p, anti-miR-21, and antimiR-199a-3p transfected neurons comparable to controls (data not shown). This suggests that there miR-21 and miR-199a-3p have similar growth effects in male and female neurons.

\section{miR-21 overexpression supports neurite growth on non-permissive substrate}

Genetic deletion of PTEN was shown to facilitate axon regeneration in the non-permissive environment of the injured CNS (Park et al., 2008; Liu et al., 2010). Thus, we asked whether the growth promoting effects of increasing miR-21 or miR-199a-3p levels affects axon growth on the non-permissive CSPG aggrecan. Naïve adult DRG neurons transfected with pre-miR-21 showed significantly increased axon growth on the aggrecan compared with 
A DRG neurons
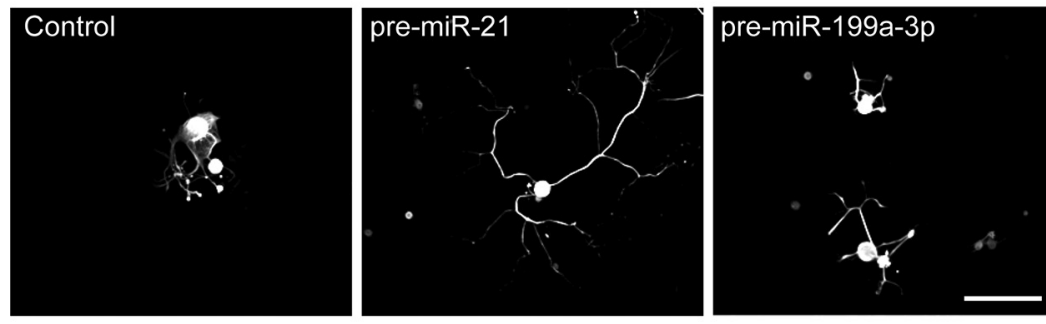

B

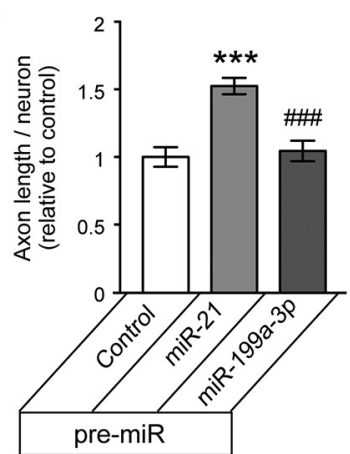

\section{Cortical neurons}

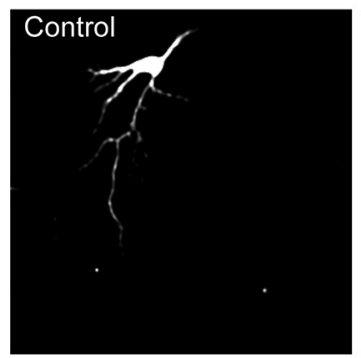

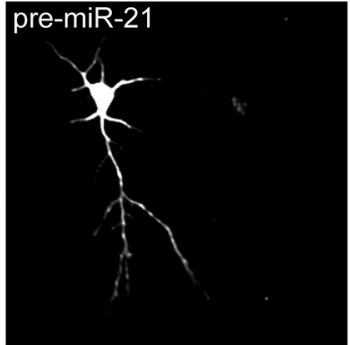

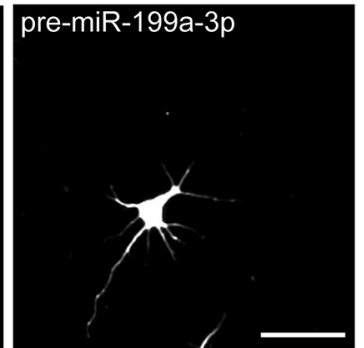

D

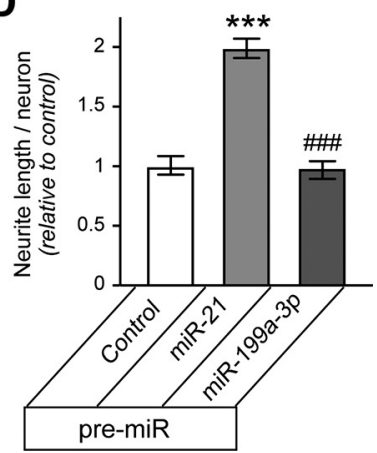

Figure 6. miR-21 overexpression supports neurite growth on non-permissive aggrecan substrate. $\boldsymbol{A}, \boldsymbol{B}$, Representative immunofluorescent images for NF in adult DRG cultures transfected with indicated pre-miRs versus scrambled RNA (control) and plated onto aggrecan (CSPG) are shown in $\boldsymbol{A}$. Total axon length per neuron is shown $\boldsymbol{B}$ as average \pm SEM relative to control. $\boldsymbol{C}$, $\boldsymbol{D}$, Representative immunofluorescent images for NF in E18 cortical neuron cultures transfected with indicated pre-miRs versus scrambled RNA and plated onto aggrecan (CSPG) are shown in $\boldsymbol{C}$. Total axon length per neuron is shown $\boldsymbol{D}$ as average \pm SEM relative to control ( $n \geq 95$ over each over 3 experimental replicates for $\boldsymbol{B}, \boldsymbol{D}$; ${ }^{* * *} p \leq 0.005$ versus control and \#\#p $\leq 0.01$ and $\# \# \# p \leq 0.005$ versus miR-21 by one-way ANOVA with pair-wise comparison and Tukey's post hoc tests). Scale bar: $100 \mu \mathrm{m}(\boldsymbol{A})$ and $50 \mu \mathrm{m}(\mathbf{C})$.

scrambled control RNA and pre-miR-199a-3p transfected neurons (Fig. 6A,B). Embryonic cortical neurons transfected with pre-miR-21 similarly showed significantly increased neurite growth on aggrecan compared the scrambled control RNA and pre-miR-199a$3 p$ transfected cultures (Fig. 6C,D). Taken together, these data suggest that increased PTEN activity contributes to neurite growth inhibition by CSPGs, with miR-21 likely able to overcome this effect by decreasing PTEN abundance.

\section{Exogenous expression of miR-resistant mTOR and PTEN protein rescues miR-mediated axon growth effects}

The data above raise the possibility that the balance of PTEN versus mTOR levels contribute to axon growth promotion and attenuation by miR-21 and miR-199a-3p, respectively. However, these experiments did not rule out contributions of other mRNA targets for the effects of miR-21 and miR-199a-3p on axon growth. To directly test whether modulation of Pten and mTor mRNA levels by these two miRs determines their effects on neurite growth, we asked whether co-transfection with miR-resistant Pten and mTor mRNAs could reverse the outcomes of miR-21 and miR-199a-3p over expression. For this, we generated PTEN and mTOR expression constructs with the predicted miR-21 and miR-199a-3p recognition sites deleted from the Pten and mTor mRNA 3' UTRs (Pten ${ }^{\Delta m i R-21}$ and mTor ${ }^{\Delta m i R-199}$, respectively). Co-transfecting Pten ${ }^{\Delta m i R-21}$ with pre-miR-21 in adult DRG neurons significantly attenuated the axon growth promotion effects seen with elevated miR-21 levels (Fig. $7 A, C$ ). Similarly, co-transfecting mTor ${ }^{\Delta m i R-199}$ with pre-miR-199a-3p in adult DRG neurons significantly reversed the axon growth attenuation seen with elevated miR-199a-3p levels (Fig. 7B,D). Considering that elevation of mTOR activity has been linked to axon growth promotion rather than $m$ Tor mRNA levels, we asked whether mTOR kinase activity is required to rescue effects of miR-199a-3p overexpression. Thus, we generated a kinase dead, miR-199a-3p-resistant mTor expression construct (mTor-Kdm ${ }^{\Delta m i R-199}$ ). In contrast to the wild-type miR-resistant mTOR, expression of mTor-Kdm ${ }^{\Delta m i R-199}$ did not mitigate effects of pre-miR199a-3p expression (Fig. 7B,D). Although we cannot completely rule out effects of other miR-21 and miR-199a-3p mRNA targets, our results indicate that depletion of Pten mRNA contributes to neurite growth promotion by miR-21 and depletion of mTor mRNA contributes to neurite growth attenuation by miR199a-3p. 

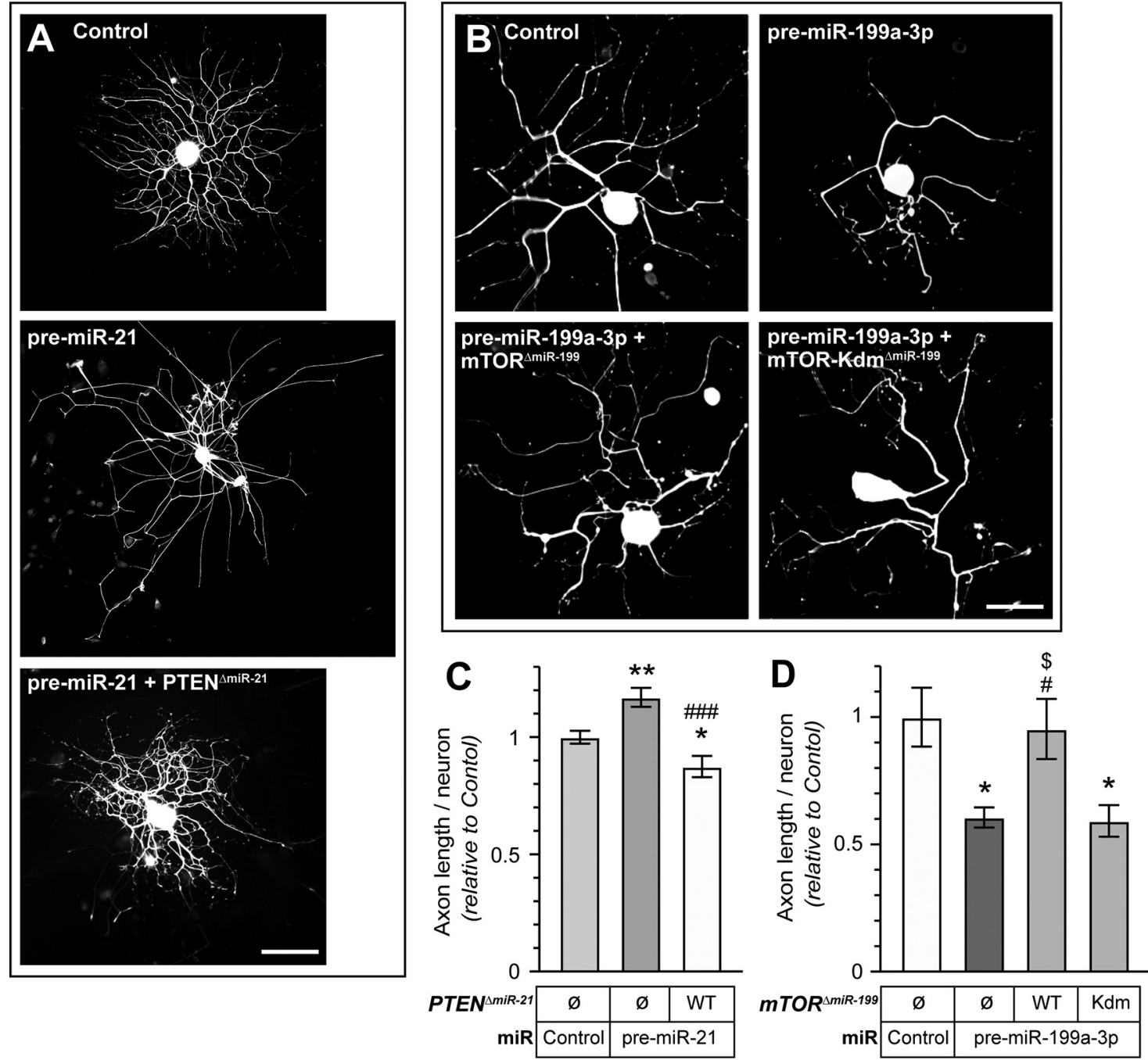

Figure 7. Exogenous $m$ Tor and Pten overcome the miR-199a-3p and miR-21 axon growth alterations. $\boldsymbol{A}, \boldsymbol{B}$, Representative immunofluorescent images for NF in adult DRG neurons co-transfected with indicated pre-miRs or scrambled RNA (control) plus expression constructs for plasmids encoding flag-tagged PTEN or mTOR with deletion of the predicted miR-199a-3p and miR-21 target sequences, respective (Pten ${ }^{\triangle m i R-21}$ and mTor $\left.{ }^{\triangle m i R-199}\right)$. Co-transfection with plasmids lacking the PTEN and mTOR coding sequences were used as control for $\boldsymbol{A}, \boldsymbol{B}$. miR-199a-3p target site deleted kinase-dead mutant mTOR with deletion of predicted miR-199a$3 p$ target sequence (mTOR-Kdm ${ }^{\triangle m i R-199}$ ) was used in $\boldsymbol{B}$. $\boldsymbol{C}, \boldsymbol{D}$, Total axon length per neuron for DRG neurons transfected as in $\boldsymbol{A}, \boldsymbol{B}$ are shown as average \pm SEM relative to control $\left(N \geq 50\right.$ neurons over 3 experimental replicates; ${ }^{*} p \leq 0.05$ and ${ }^{* *} p \leq 0.01$ vs control, $\# p \leq 0.05$ and \#\#\#p $\leq 0.005$ vs miR-21, and $\$ p \leq 0.05$ vs mTOR-Kdm by one-way ANOVA with pair-wise comparison and Tukey's post hoc tests). Scale bars: $100 \mu \mathrm{m}(\boldsymbol{A}, \boldsymbol{B})$.

\section{Levels of miR-21 and miR-199a-3p affect axonal protein synthesis in DRG neurons}

Increase in mTOR activity is well established to increase protein synthesis through downstream modifications in activity of translation factors (Switon et al., 2017). Notably, work in the CNS injury models where PTEN gene is deleted or PTEN protein is inhibited have largely focused on downstream activation of S6 kinase (S6K) or phosphorylation of ribosomal protein S6 (rpS6) to indicate increased mTOR activity, rather than activation of neuronal protein synthesis (Park et al., 2008; Liu et al., 2010; Ohtake et al., 2014; Urban et al., 2019; Walker et al., 2019). Moreover, a novel pharmacological approach for neurite growth modulators indicated that activity of S6K can impede rather than support neurite growth (Al-Ali et al., 2017). Thus, we asked whether depletion of Pten or mTor mRNAs by overexpression of miR-21 and miR199a-3p impact neuronal protein synthesis. We initially used a puromycinylation assay to quantify translation of endogenous mRNAs in the DRG cultures. DRG cultures transfected with pre-miR-21 showed significantly higher puromycin incorporation in the neuronal cell bodies than control RNA or pre-miR-199a-3p transfected neurons (Fig. 8A,B). This increased puromycin incorporation also occurred in the axons of the pre-miR-21 transfected neurons, while the pre-miR-199a-3p transfected neurons showed modest but significantly decreased puromycin incorporation (Fig. $8 A, B$ ). These data indicate that elevation 
A

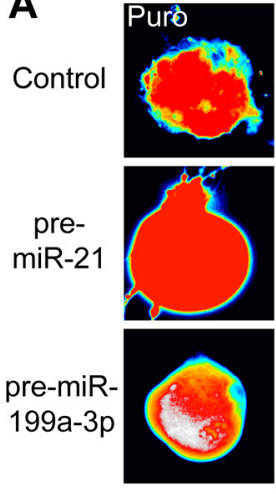

C

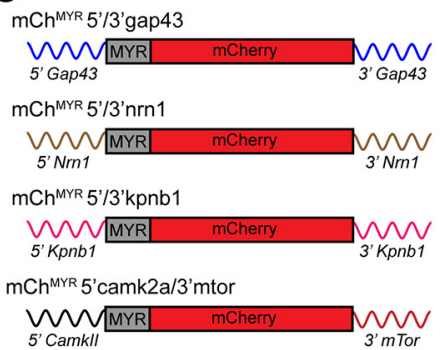

E $\mathrm{mCh}^{\text {MYR }} 5^{\prime} / 3^{\prime}$ gap43

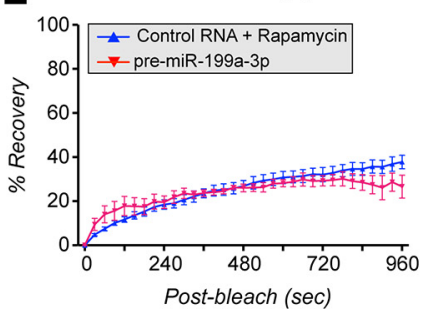

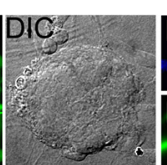
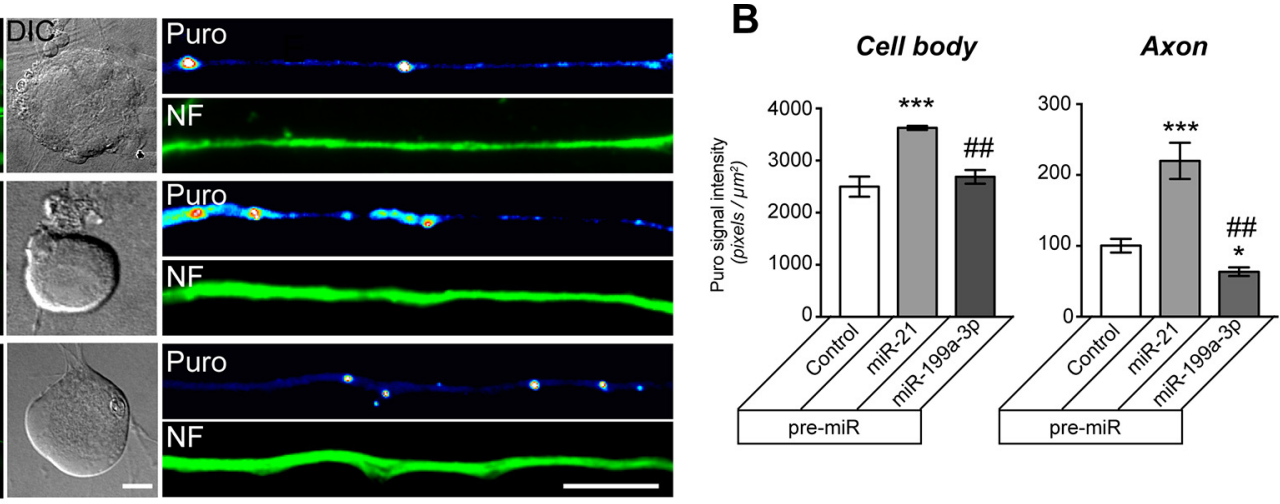

D
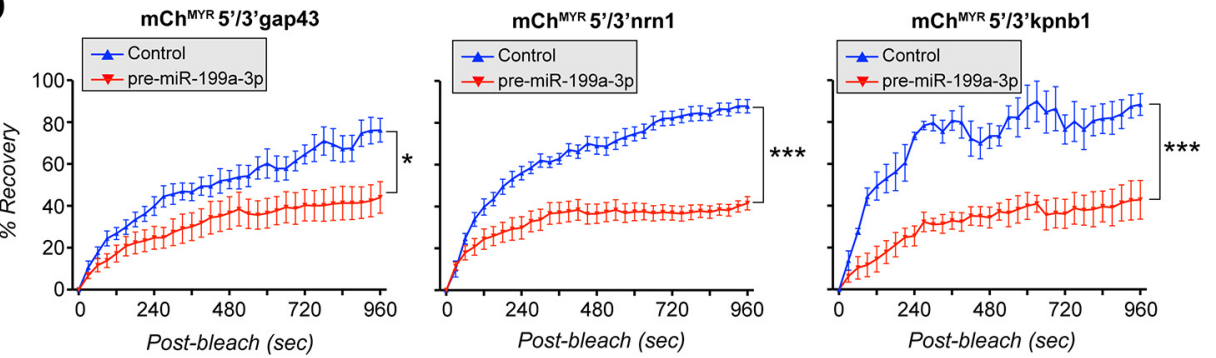

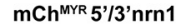
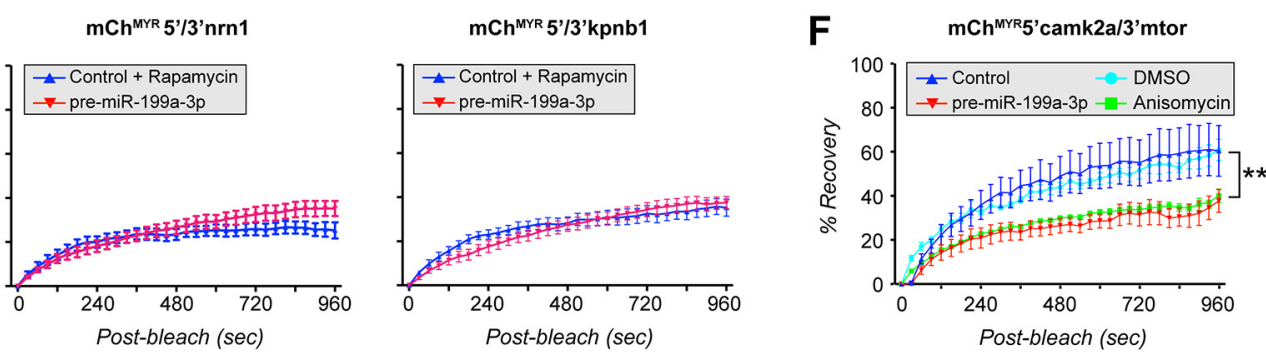

Figure 8. miR-199a-3p and miR-21 modulate neuronal protein synthesis. $\boldsymbol{A}, \boldsymbol{B}$, Representative fluorescent images for puromycin (Puro) incorporation in DRG neurons transfected with indicated pre-miRs or scrambled RNA (control) and treated with OPP are shown for cell bodies (left three columns) distal axons (right column). OPP incorporation was detected by labeling with Alexa Fluor 594 using the Click chemistry. $\boldsymbol{B}$, Average puromycin incorporation in cell bodies and axons $(N \geq 50$ axons each over 3 experimental replicates; ${ }^{*} p \leq 0.001,{ }^{* \star *} p \leq 0.001$ vs control and \#\#p $\leq 0.005$ vs miR-21 by one-way ANOVA with pairwise comparison and Tukey's HSD post hoc tests). Scale bars: $10 \mu \mathrm{m}$. C, Schematics of intra-axonal translation reporter constructs used for FRAP experiments for panels $\boldsymbol{D}-\boldsymbol{F}$ and Extended Data Figure 8-1. $\boldsymbol{D}, \boldsymbol{E}$, Quantitation of FRAP sequences for distal axons of DRGs expressing $m C h^{M Y R} 5^{\prime} / 3^{\prime} g a p 43, m C h^{M Y R} 5^{\prime} / 3^{\prime} n r n 1$, or $m C h^{M Y R} 5^{\prime} / 3^{\prime} k p n b 1$ mRNAs that were cotransfected with pre-miR-199a-3p or scrambled RNA are shown. Axonal mCherry fluorescent recovery for each reporter was significantly attenuated by miR-199a-3p overexpression. Values shown as normalized average \% recovery \pm SEM; representative image sequences shown in Extended Data Figure 8-1. E, FRAP analysis for $m C h^{M Y R} 5^{\prime} / 3^{\prime}$ gap 43, $m C h^{M Y R} 5^{\prime} / 3^{\prime} n r n 1$, or $m C h^{M Y R} 5^{\prime} / 3^{\prime} k p n b 1$ mRNA expressing DRG neurons that were transfected with scrambled RNA and treated with rapamycin $2 \mathrm{~h}$ before FRAP show diminished fluorescence recovery comparable to miR-199a-3p overexpression. Values shown as normalized average \% recovery $\pm \mathrm{SEM}$; representative image sequences shown in Extended Data Figure 8-1. F, FRAP analyses for distal axons of $m C h^{M Y R} 5^{\prime}$ camkll/3'mtor mRNA expressing adult DRG transfected with either pre-miR-199a-3p or scrambled RNA are shown. Data are shown as normalized average \% recovery \pm SEM and representative image sequences shown in Extended Data Figure 8-1. Scrambled RNA transfected neurons were also analyzed after treatment with anisomycin or vehicle control (DMSO), with the anisomycin-treated neurons indicating translation dependence for the recovery ( $N \geq 13$ axons over $\geq 3$ experimental replicates for $\boldsymbol{D}-\boldsymbol{F} ;{ }^{*} p \leq 0.01,{ }^{* *} p \leq 0.005,{ }^{* \star *} p \leq 0.001$ by one-way ANOVA with pairwise comparison and Tukey's HSD post hoc tests).

of miR-21 increases both overall and intra-axonal protein synthesis, while elevation of miR-199a-3p has the opposite effect.

Previous studies have indicated that $m$ Tor mRNA can be translated in distal axons, both in cultured neurons and in vivo (Kye et al., 2014; Terenzio et al., 2018), so it is intriguing that pre-miR-199a-3p transfection reduced axonal puromycinylation. Thus, we asked whether pre-
miR-199a-3p transfection would affect translation of axonal mRNAs whose protein products have been linked to axon regeneration. We focused specifically on Importin $\beta 1$ (Kpnb1), Neuritin 1 (Nrn1), and Growth-associated protein 43 (Gap43) mRNAs whose $5^{\prime}$ and 3' UTRs have been shown to support axonal localization and translation of their mRNAs in DRG neurons using fluorescent reporters (Merianda et al., 2013; Yoo et al., 2013; Perry et al., 
2016; Sahoo et al., 2018, 2020; Terenzio et al., 2018). We used a FRAP assay in DRG cultures co-expressing premiR-199a-3p plus mCherry ${ }^{\mathrm{Mr}}$ ( $\mathrm{mCh}^{\mathrm{MYR}}$ ) reporters with the $5^{\prime}$ and $3^{\prime}$ UTRs of Kpnb1, Nrn1, or Gap43 mRNAs (mCh ${ }^{\mathrm{MYR}_{5}}$ '/3'gap43, mCh ${ }^{\mathrm{MYR}} 5^{\prime} / 3^{\prime} \mathrm{kpnb}^{\prime}$, and $\mathrm{mCh}^{\mathrm{MYR}_{5}}$ '/ 3'nrn1; Fig. 8C). Axonal recovery after photobleaching in transfected DRGs was previously shown to be translation-dependent for each of these reporters (Sahoo et al., $2018,2020)$. Expression of pre-miR-199a-3p significantly decreased recovery of photobleached $\mathrm{mCh}^{\mathrm{MYR}_{5}}$ '/ 3'gap43, mCh ${ }^{\mathrm{MYR}} 5^{\prime} / 3^{\prime} \mathrm{kpnb}$, and $\mathrm{mCh}^{\mathrm{MYR}} 5^{\prime} / 3^{\prime} \mathrm{nrn} 1$ in distal axons of the cultured DRG neurons (Fig. 8D). Moreover, in control RNA transfected cultures, axon pretreatment with the mTOR inhibitor rapamycin before photobleaching decreased recovery of $\mathrm{mCh}^{\mathrm{MYR}_{5}}$ ' $^{\prime}$ ' $^{\prime}$ gap43,

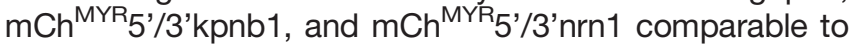
the recovery seen in miR-199a-3p overexpressing neurons (Fig. 8E). We next asked whether overexpression of miR199a-3p would affect intra-axonal translation of $m$ Tor mRNA. For this we generated an $\mathrm{mCh}^{\mathrm{MYR}}$ reporter with the $3^{\prime}$ UTR of $m$ Tor mRNA containing the predicted miR-199a$3 p$ target sequence and the $5^{\prime}$ UTR of Camk2a mRNA (mCh ${ }^{\mathrm{MY}}{ }_{5}$ 'camk2a/3'mtor; Fig. 8C; Extended Data Fig. 8$1 A-C)$. The $m$ Tor $3^{\prime}$ UTR is sufficient for axonal localization (Terenzio et al., 2018), while the Camk2a supports translation in axons but has no axonal localizing activity (Merianda et al., 2013). FRAP for the mCh ${ }^{\mathrm{MYR}} 5$ 'camk2a/3'mtor transfected DRG axons showed that cotransfection with premiR-199a-3p reduced fluorescence recovery comparable to what was seen with the protein synthesis inhibitor anisomycin (Fig. 8F; Extended Data Fig. 8-1D). Taken together, these data indicate that manipulation of neuronal PTEN and mTOR activity through posttranscriptional mechanisms change both overall and intraaxonal protein synthesis.

\section{Discussion}

Here, we show that miR-21 promotes axon growth from adult DRG neurons and neurite growth from embryonic cortical neurons, while miR-199a-3p does the opposite. Consistent with the increased axonal growth seen after peripheral nerve injury conditioning, miR-21 levels are increased in injury-conditioned DRG neurons while levels of miR-199a-3p decreased. Importantly, we show that miR21 binds to and depletes Pten mRNA and miR-199a-3p binds to and depletes $m$ Tor mRNA in adult neurons, with commensurate decreases in the proteins encoded by these mRNAs. Furthermore, the growth promoting effects of miR-21 and inhibiting effects of miR-199a-3p are completely reversed by introduction of Pten and $m$ Tor mRNAs lacking the corresponding target sites for these miRs in their 3' UTRs. While these miRs undoubtedly have many mRNA targets, including several with roles in neurite growth that have been recently published (Li et al., 2020; Liu et al., 2020; Song et al., 2020; Laliberte et al., 2021; Nakashima et al., 2021), our findings emphasize that depletion of PTEN through increased miR-21 increases axon growth and depletion of mTOR through increased miR199a-3p decreases axon growth.
Several studies have linked alterations in miR levels, including miR-21, to promotion of axon growth and regeneration in the PNS and CNS. For example, co-transfection of miR-21 and miR-338 into rat spinal cord neurons increased regeneration after sciatic nerve injury (Wang et al., 2016). Lopez-Leal et al. (2020) recently reported that cultures of denervated Schwann cells secrete miR-21 in exosomes, and those exosomes decrease PTEN levels and increase axon outgrowth when applied to DRG explant cultures (Lopez-Leal et al., 2020). Exosomes isolated from mesenchymal stem cells and differentiated PC12 cells that contain miR-21 and miR-19b were also shown to increase recovery from $\mathrm{SCl}$ in rodents (Xu et al., 2019). Further exercise-induced miR-21 in the injured spinal cord is also packaged into exosomes (Li et al., 2020). However, there are other reports that increased miR-21 in the injured spinal cord correlate with poor recovery (Chung et al., 2020), and knock-down of miR-21 improved recovery after $\mathrm{SCl}$ with alleviation of peripheral inflammatory cytokine expression and BDNF elevation in the injured spinal cord (Xie et al., 2018). The basis of these data that conflict with previous work showing elevated miR-21 with exercise after $\mathrm{SCl}$ is not clear, and our data unequivocally show that increasing neuronal miR-21 increases axon growth, including growth on the non-permissive CSPG substrate aggrecan.

Our data indicate that increased miR-199a-3p expression reduces $m$ Tor $\mathrm{mRNA}$ translation in axons and results in decreased translation of axonal mRNAs encoding proteins linked to an increase in PNS axon regeneration. Although it is not clear whether miR-199a-3p localizes into injured spinal cord axons as we see with the DRG cultures here, our data suggest that the $\mathrm{SCl}$-induced increase miR-199a-3p reported by Liu et al. (2012) could decrease capacity for axon regeneration in those animals. Similarly, the reported exercise-induced increase in miR21 and decrease in miR-199a-3p after SCl (Liu et al., 2012) could shift the injured neurons to a fate favorable for axon regeneration. Consistent with this, exercise of rats post-SCl has been shown to increase the number of sensory axons regenerating into a peripheral nerve grafted into the transected spinal cord (Sachdeva et al., 2016). Further, work from Li et al. (2020) recently showed that exercise-associated miR-21 increase correlates with increased functional recovery from $\mathrm{SCl}$ with the elevated miR-21 targeting programmed cell death protein 4 (PCD4; $\mathrm{Li}$ et al., 2020). In some paradigms, exercise has also been shown to increase axon regeneration after peripheral nerve injury (Sabatier and English, 2015; Cannoy et al., 2016). Furthermore, DRG neurons cultured from adult rats that have undergone voluntary exercise showed increased in vitro axon growth that statistically correlated with the distance the animals had run (Molteni et al., 2004). These data suggest that the increase in miR-21 and decrease in miR-199a-3p detected in the injury-conditioned DRG neurons here could indeed contribute to ability faster axon extension seen in those neurons. However, it should be noted that miR-21 and miR-199a$3 p$ do not always show reciprocal regulation as seen here, since both miRs were decreased in DRGs from diabetic mice (Cheng et al., 2015). 
Many studies have now shown that the PTEN/mTOR pathway can alter growth potential of neurons, with increased mTOR activity supporting axon regeneration in both the CNS and PNS (Park et al., 2008; Abe et al., 2010; Liu et al., 2010; Sun et al., 2011). Phosphorylation of S6K and RP S6 have often been taken as evidence for increased mTOR activity and the resulting increased mRNA translation in these axon injury model systems (Park et al., 2008; Abe et al., 2010; Liu et al., 2010; Sun et al., 2011; Urban et al., 2019). Notably, although phosphorylated RP S6 tends to be associated with translationally active ribosomes, the phosphorylation status of RP S6 does not always reflect a change in protein synthesis (Biever et al., 2015). Recent work using S6K inhibitors indicates that S6K activation can attenuate CNS axon regeneration (AlAli et al., 2017). Our data show that altering the balance of PTEN/mTOR levels in neurons does indeed alter neuronal protein synthesis, including translation through Kpnb1, Nrn1, and Gap43 mRNA UTRs in axons. This is consistent with recent work pointing to loss of axonal protein synthesis when mTor mRNA's localization into sciatic nerve axons was attenuated through deletion of its $3^{\prime}$ UTR (Terenzio et al., 2018; Sahoo et al., 2020).

The increase in miR-21 and decrease in miR-199a-3p levels in injury-conditioned neurons is consistent with previous work pointing to posttranscriptional regulation of axon growth from injury-conditioned DRG neurons (Smith and Skene, 1997; Twiss et al., 2000). Our data suggest that miR-21 is at saturating levels in both the injury-conditioned DRG neurons and embryonic cortical neurons, as miR-21 overexpression did not further increase neurite growth in either preparation. However, overexpression of miR-199a-3p was able to decrease neurite growth in both neuronal populations indicating that depleting $m T$ Tor mRNA from these neurons can overcome effects of saturating miR-21 levels. PNS neurons have been hypothesized to switch to an embryonic gene expression program that supports axon growth after injury, and recent profiling of ribosome bound mRNAs in corticospinal neurons after $\mathrm{SCl}$ indicates that these CNS neurons similarly shift to an embryonic gene expression program, with regeneration promoting stem cell grafts causing sustained expression of the growth-associated genes (Poplawski et al., 2020). Our observations that miR-21 is saturated for growth on permissive substrates in both the injury-conditioned adult DRG and embryonic cortical neurons raise the possibility that posttranscriptional regulation of gene expression may also shift toward a more embryonic state to support growth programs through modulation of PTEN to mTOR pathway. PTEN can modify activity of other downstream targets beyond mTOR, and increased neurite growth in murine DRG cultures after inhibition or depletion of PTEN was shown to be insensitive to mTOR inhibition with rapamycin (Cheng et al., 2015). However, rapamycin-resistant mTOR activity was more recently reported to support regeneration of injury-conditioned DRG axons, both in vivo after SCl and in culture (Chen et al., 2016). Consistent with this, localized mTOR activity supports regeneration of injured PNS axons through its translation promoting activity (Terenzio et al., 2018; Sahoo et al., 2020).
The Court lab recently reported that transition of Schwann cells to a repair phenotype results in secretion of exosomes containing miR-21. Similar to our data, they reported increased axonal growth with depletion of Pten mRNA from in cultured DRG neurons exposed to these Schwann cell-derived exosomes (Lopez-Leal et al., 2020). Since the DRG cultures used here contain Schwann cells that have lost contact with axons, we cannot exclude that some of the changes in miR-21 levels derive from Schwann cells. Nonetheless, our data emphasize a direct effect of miR-21 on neuronal PTEN expression and we see that endogenous miR-21 and miR-199a-3p localize into axons of the cultured DRG neurons.

miRs have been detected in axons of many different neuronal populations with numerous functional effects seen with overexpression or depletion of these miRs. To our knowledge, this is the first report of axonal miRs directly targeting Pten and $m$ Tor mRNAs that subsequently regulate translation of other mRNAs, including mRNAs localizing into growing axons. Interestingly, our manipulations of the PTEN/mTOR pathway and resulting effects on protein synthesis by overexpression and inhibition of miR21 and miR-199a-3p selectively affected axon elongation rather than axon branching. Transcriptional programs associated with sprouting or branching of sensory neurons have been reported for both in vivo and cultured neurons (Harrison et al., 2015; Lemaitre et al., 2020). In contrast to these findings, our data emphasize that the elongating axon growth regulated by the balance of miR21 and miR-199a-3p levels is impacted by direct posttranscriptional regulation of Pten and mTor mRNAs.

\section{References}

Abe N, Borson SH, Gambello MJ, Wang F, Cavalli V (2010) mTOR activation increases axonal growth capacity of injured peripheral nerves. J Biol Chem 285:28034-28043.

Al-Ali H, Ding $Y$, Slepak T, Wu W, Sun $Y$, Martinez $Y, X u X M$, Lemmon VP, Bixby JL (2017) The mTOR substrate S6 kinase 1 (S6K1) is a negative regulator of axon regeneration and a potential drug target for central nervous system injury. J Neurosci 37:7079-7095.

Bae B, Miura P (2020) Emerging roles for 3' UTRs in neurons. Int J Mol Sci 21:3413.

Biever A, Valjent E, Puighermanal E (2015) Ribosomal protein S6 phosphorylation in the nervous system: from regulation to function. Front Molec Neurosci 8:75.

Cannoy J, Crowley S, Jarratt A, Werts KL, Osborne K, Park S, English AW (2016) Upslope treadmill exercise enhances motor axon regeneration but not functional recovery following peripheral nerve injury. J Neurophysiol 116:1408-1417.

Chen W, Lu N, Ding Y, Wang Y, Chan LT, Wang X, Gao X, Jiang S, Liu K (2016) Rapamycin-resistant mTOR activity is required for sensory axon regeneration induced by a conditioning lesion. eNeuro 3:ENEURO.0358-0316.2016.

Cheng C, Kobayashi M, Martinez JA, Ng H, Moser JJ, Wang X, Singh V, Fritzler MJ, Zochodne DW (2015) Evidence for epigenetic regulation of gene expression and function in chronic experimental diabetic neuropathy. J Neuropathol Exp Neurol 74:804-817.

Christie KJ, Webber CA, Martinez JA, Singh B, Zochodne DW (2010) PTEN inhibition to facilitate intrinsic regenerative outgrowth of adult peripheral axons. J Neurosci 30:9306-9315.

Chung HJ, Chung WH, Do SH, Lee JH, Kim HY (2020) Up-regulation of microRNAs-21 and -223 in a Sprague-Dawley rat model of traumatic spinal cord injury. Brain Sci 10:141. 
Côté MP, Azzam GA, Lemay MA, Zhukareva V, Houlé JD (2011) Activity-dependent increase in neurotrophic factors is associated with an enhanced modulation of spinal reflexes after spinal cord injury. J Neurotrauma 28:299-309.

Craig A, Banker G (1994) Neuronal polarity. Annu Rev Neurosci 17:267-310.

Dalla Costa I, Buchanan C, Zdradzinski MD, Sahoo PK, Smith TP, Thames E, Kar AN, Twiss JL (2021) The functional organization of axonal mRNA transport and translation. Nat Rev Neurosci 22:7791.

Duan X, Qiao M, Bei F, Kim IJ, He Z, Sanes JR (2015) Subtype-specific regeneration of retinal ganglion cells following axotomy: effects of osteopontin and mTOR signaling. Neuron 85:1244-1256.

Harrison BJ, Venkat G, Hutson T, Rau KK, Bunge MB, Mendell LM, Gage FH, Johnson RD, Hill C, Rouchka EC, Moon L, Petruska JC (2015) Transcriptional changes in sensory ganglia associated with primary afferent axon collateral sprouting in spared dermatome model. Genom Data 6:249-252.

Kim HH, Kim P, Phay M, Yoo S (2015) Identification of precursor microRNAs within distal axons of sensory neuron. $\mathrm{J}$ Neurochem 134:193-199.

Kye MJ, Niederst ED, Wertz MH, Gonçalves Ido C, Akten B, Dover KZ, Peters M, Riessland M, Neveu P, Wirth B, Kosik KS, Sardi SP, Monani UR, Passini MA, Sahin M (2014) SMN regulates axonal local translation via miR-183/mTOR pathway. Hum Mol Genet 23:6318-6331.

Laliberte AM, Karadimas SK, Vidal PM, Satkunendrarajah K, Fehlings MG (2021) Mir21 modulates inflammation and sensorimotor deficits in cervical myelopathy: data from humans and animal models. Brain Commun 3:fcaa234.

Lemaitre D, Hurtado ML, De Gregorio C, Oñate M, Martínez G, Catenaccio A, Wishart TM, Court FA (2020) Collateral sprouting of peripheral sensory neurons exhibits a unique transcriptomic profile. Mol Neurobiol 57:4232-4249.

Li M, Jiang WT, Li J, Ji WC (2020) Exercise protects against spinal cord injury through miR-21-mediated suppression of PDCD4. Am J Transl Res 12:5708-5718.

Liu G, Detloff MR, Miller KN, Santi L, Houlé JD (2012) Exercise modulates microRNAs that affect the PTEN/mTOR pathway in rats after spinal cord injury. Exp Neurol 233:447-456.

Liu J, Zhang S, Huang Y, Sun L (2020) miR-21 protects neonatal rats from hypoxic-ischemic brain damage by targeting CCL3. Apoptosis 25:275-289.

Liu K, Lu Y, Lee JK, Samara R, Willenberg R, Sears-Kraxberger I, Tedeschi A, Park KK, Jin D, Cai B, Xu B, Connolly L, Steward O, Zheng B, He Z (2010) PTEN deletion enhances the regenerative ability of adult corticospinal neurons. Nat Neurosci 13:1075-1081.

Lopez-Leal R, Diaz-Viraque F, Catalan RJ, Saquel C, Enright A, Iraola G, Court FA (2020) Schwann cell reprogramming into repair cells increases exosome-loaded miRNA-21 promoting axonal growth. J Cell Sci 133:jcs239004.

Mendell LM, Munson JB, Arvanian VL (2001) Neurotrophins and synaptic plasticity in the mammalian spinal cord. J Physiol 533:91-97.

Merianda TT, Gomes C, Yoo S, Vuppalanchi D, Twiss JL (2013) Axonal localization of neuritin/CPG15 mRNA in neuronal populations through distinct 5 ' and 3' UTR elements. J Neurosci 33:13735-13742.

Molteni R, Zheng JQ, Ying Z, Gómez-Pinilla F, Twiss JL (2004) Voluntary exercise increases axonal regeneration from sensory neurons. Proc Natl Acad Sci USA 101:8473-8478.

Nakashima $\mathrm{H}$, Tsujimura $\mathrm{K}$, Irie $\mathrm{K}$, Imamura T, Trujillo CA, Ishizu M, Uesaka M, Pan M, Noguchi H, Okada K, Aoyagi K, Andoh-Noda T, Okano H, Muotri AR, Nakashima K (2021) MeCP2 controls neural stem cell fate specification through miR-199a-mediated inhibition of BMP-Smad signaling. Cell Rep 35:109124.

Neumann S, Woolf CJ (1999) Regeneration of dorsal column fibers into and beyond lesion site following adult spinal cord injury. Neuron 23:83-91.
Neumann S, Bradke F, Tessier-Lavigne M, Basbaum Al (2002) Regeneration of sensory axons within injured spinal cord induced by intraganglionic cAMP elevation. Neuron 34:885-893.

Ohtake Y, Park D, Abdul-Muneer PM, Li H, Xu B, Sharma K, Smith GM, Selzer ME, Li S (2014) The effect of systemic PTEN antagonist peptides on axon growth and functional recovery after spinal cord injury. Biomaterials 35:4610-4626.

Park KK, Liu K, Hu Y, Smith PD, Wang C, Cai B, Xu B, Connolly L, Kramvis I, Sahin M, He Z (2008) Promoting axon regeneration in the adult CNS by modulation of the PTEN/mTOR pathway. Science 322:963-966.

Perry RBT, Rishal I, Doron-Mandel E, Kalinski AL, Medzihradszky KF, Terenzio M, Alber S, Koley S, Lin A, Rozenbaum M, Yudin D, Sahoo PK, Gomes C, Shinder V, Geraisy W, Huebner EA, Woolf CJ, Yaron A, Burlingame AL, Twiss JL, et al. (2016) Nucleolinmediated RNA localization regulates neuron growth and cycling cell size. Cell Rep 16:1664-1676.

Phay M, Kim HH, Yoo S (2015) Dynamic change and target prediction of axon-specific microRNAs in regenerating sciatic nerve. PLoS One 10:e0137461.

Poplawski GHD, Kawaguchi R, Van Niekerk E, Lu P, Mehta N, Canete P, Lie R, Dragatsis I, Meves JM, Zheng B, Coppola G, Tuszynski MH (2020) Injured adult neurons regress to an embryonic transcriptional growth state. Nature 581:77-82.

Popovitchenko T, Rasin MR (2017) Transcriptional and post-transcriptional mechanisms of the development of neocortical lamination. Front Neuroanat 11:102.

Rishal I, Golani O, Rajman M, Costa B, Ben-Yaakov K, Schoenmann Z, Yaron A, Basri R, Fainzilber M, Galun M (2013) WIS-NeuroMath enables versatile high throughput analyses of neuronal processes. Dev Neurobiol 73:247-256.

Sabatier MJ, English AW (2015) Pathways mediating activity-induced enhancement of recovery from peripheral nerve injury. Exerc Sport Sci Rev 43:163-171.

Sachdeva R, Theisen CC, Ninan V, Twiss JL, Houlé JD (2016) Exercise dependent increase in axon regeneration into peripheral nerve grafts by propriospinal but not sensory neurons after spinal cord injury is associated with modulation of regeneration-associated genes. Exp Neurol 276:72-82.

Sahoo PK, Lee SJ, Jaiswal PB, Alber S, Kar AN, Miller-Randolph S, Taylor EE, Smith T, Singh B, Ho TSY, Urisman A, Chand S, Pena EA, Burlingame AL, Woolf CJ, Fainzilber M, English AW, Twiss JL (2018) Axonal G3BP1 stress granule protein limits axonal mRNA translation and nerve regeneration. Nat Commun 9:3358.

Sahoo PK, Kar AN, Samra N, Terenzio M, Patel P, Lee SJ, Miller S, Thames E, Jones B, Kawaguchi R, Coppola G, Fainzilber M, Twiss $\mathrm{JL}$ (2020) A translational switch drives axonal stress granule disassembly through casein kinase $2 \alpha$. Curr Biol 30:4882-4895.E6.

Smith DS, Skene P (1997) A transcription-dependent switch controls competence of adult neurons for distinct modes of axon growth. $J$ Neurosci 17:646-658.

Smith TP, Sahoo PK, Kar AN, Twiss JL (2020) Intra-axonal mechanisms driving axon regeneration. Brain Res 1740:146864.

Song D, Li G, Hong Y, Zhang P, Zhu J, Yang L, Huang J (2020) miR199a decreases neuritin expression involved in the development of Alzheimer's disease in APP/PS1 mice. Int $\mathrm{J}$ Mol Med 46:384396.

Sun F, Park KK, Belin S, Wang D, Lu T, Chen G, Zhang K, Yeung C, Feng G, Yankner BA, He Z (2011) Sustained axon regeneration induced by co-deletion of PTEN and SOCS3. Nature 480:372-375.

Switon K, Kotulska K, Janusz-Kaminska A, Zmorzynska J, Jaworski $J(2017)$ Molecular neurobiology of mTOR. Neuroscience 341:112153.

Terenzio M, Koley S, Samra N, Rishal I, Zhao Q, Sahoo PK, Urisman A, Marvaldi L, Oses-Prieto JA, Forester C, Gomes C, Kalinski AL, Di Pizio A, Doron-Mandel E, Perry RB, Koppel I, Twiss JL, Burlingame AL, Fainzilber M (2018) Locally translated mTOR controls axonal local translation in nerve injury. Science 359:14161421. 
Twiss JL, Smith DS, Chang B, Shooter EM (2000) Translational control of ribosomal protein L4 mRNA is required for rapid neurite regeneration. Neurobiol Dis 7:416-428.

Urban MW, Ghosh B, Block CG, Strojny LR, Charsar BA, Goulão M, Komaravolu SS, Smith GM, Wright MC, Li S, Lepore AC (2019) Long-distance axon regeneration promotes recovery of diaphragmatic respiratory function after spinal cord injury. eNeuro 6: ENEURO.0096-0019.2019.

Vuppalanchi D, Coleman J, Yoo S, Merianda TT, Yadhati AG, Hossain J, Blesch A, Willis DE, Twiss JL (2010) Conserved 3'-untranslated region sequences direct subcellular localization of chaperone protein mRNAs in neurons. J Biol Chem 285:18025-18038.

Vuppalanchi D, Merianda TT, Donnelly C, Pacheco A, Williams G, Yoo S, Ratan RR, Willis DE, Twiss JL (2012) Lysophosphatidic acid differentially regulates axonal mRNA translation through 5'UTR elements. Mol Cell Neurosci 50:136-146.

Walker CL, Wu X, Liu NK, Xu XM (2019) Bisperoxovanadium mediates neuronal protection through inhibition of PTEN and activation of PI3K/AKT-mTOR signaling after traumatic spinal injuries. J Neurotrauma 36:2676-2687.

Wang J, Muheremu A, Zhang M, Gong K, Huang C, Ji Y, Wei Y, Ao Q (2016) MicroRNA-338 and microRNA-21 co-transfection for the treatment of rat sciatic nerve injury. Neurol Sci 37:883-890.

Willis DE, Twiss JL (2010) Regulation of protein levels in subcellular domains through mRNA transport and localized translation. Mol Cell Proteomics 9:952-962.
Xie W, Yang SY, Zhang Q, Zhou Y, Wang Y, Liu R, Wang W, Shi J, Ning B, Jia T (2018) Knockdown of microRNA-21 promotes neurological recovery after acute spinal cord injury. Neurochem Res 43:1641-1649.

Xu G, Ao R, Zhi Z, Jia J, Yu B (2019) miR-21 and miR-19b delivered by $\mathrm{hMSC}$-derived $\mathrm{EV}$ s regulate the apoptosis and differentiation of neurons in patients with spinal cord injury. J Cell Physiol 234:10205-10217.

Ying Z, Roy RR, Edgerton VR, Gómez-Pinilla F (2005) Exercise restores levels of neurotrophins and synaptic plasticity following spinal cord injury. Exp Neurol 193:411-419.

Yoo S, Kim HH, Kim P, Donnelly CJ, Kalinski AL, Vuppalanchi D, Park M, Lee SJ, Merianda TT, Perrone-Bizzozero NI, Twiss JL (2013) A HuD-ZBP1 ribonucleoprotein complex localizes GAP43 mRNA into axons through its 3' untranslated region AU-rich regulatory element. J Neurochem 126:792-804.

Yudin D, Hanz S, Yoo S, lavnilovitch E, Willis D, Gradus T, Vuppalanchi D, Segal-Ruder Y, Ben-Yaakov K, Hieda M, Yoneda Y, Twiss JL, Fainzilber M (2008) Localized regulation of axonal RanGTPase controls retrograde injury signaling in peripheral nerve. Neuron 59:241-252.

Zheng JQ, Kelly TK, Chang B, Ryazantsev S, Rajasekaran AK, Martin KC, Twiss JL (2001) A functional role for intra-axonal protein synthesis during axonal regeneration from adult sensory neurons. $J$ Neurosci 21:9291-9303. 